\title{
Spinal ganglia control nociceptive input to the central nervous system
}

Han Hao', Rosmaliza Ramli²,3, Caixue Wang ${ }^{1}$, Chao Liư ${ }^{4}$, Shihab Shah², Pierce Mullen², Varinder Lall², Frederick Jones ${ }^{2}$, Jicheng Shao', Hailin Zhang ${ }^{1}$, David B. Jaffe ${ }^{5}$, Nikita Gamper ${ }^{1,2 *}$, Xiaona Du ${ }^{1 *}$

1Department of Pharmacology, Hebei Medical University; The Key Laboratory of Neural and Vascular Biology, Ministry of Education, China; The Key Laboratory of New Drug Pharmacology and Toxicology, Hebei Province; Shijiazhuang 050011 China.

${ }^{2}$ Faculty of Biological Sciences, University of Leeds, Leeds LS2 9JT, UK.

${ }^{3}$ School of Dental Sciences, Universiti Sains Malaysia, Kelantan 16150, Malaysia.

${ }^{4}$ Department of Animal care, Hebei Medical University; The Key Laboratory of Experimental Animal, Hebei Province; Shijiazhuang, 050011 China.

${ }^{5}$ Department of Biology, UTSA Neurosciences Institute, University of Texas at San Antonio, San Antonio, Texas, 78249, USA

*Correspondence to: Xiaona Du (uu xiaona@hotmail.com) or Nikita Gamper (‥gamper@leeds.ac.uk)

Keywords: Dorsal root ganglia; GABA; GABAergic neuron precursor cell; Pain; Sensory neuron 


\begin{abstract}
:
Accumulating observations suggest that peripheral somatosensory ganglia may regulate pain transmission, yet direct evidence is sparse. Here we show that the peripheral afferent nociceptive information undergoes dynamic filtering within dorsal root ganglia (DRG) and suggest that this filtering occurs at the axonal bifurcations (t-junctions). Using synchronous in vivo electrophysiological recordings in the peripheral (spinal nerve) and central (dorsal root) processes of the sensory nerve, ganglionic transplantation of GABAergic progenitor cells, and optogenetics we demonstrate tonic and dynamic filtering of action potentials traveling through the DRG. Filtering induced by focal application of GABA or optogenetic GABA release from the DRGtransplanted GABAergic progenitor cells was specific to nociceptive fibers. Light-sheet imaging and computer modeling demonstrated that, compared to other somatosensory fiber types, nociceptors have shorter stem axons, making somatic control over t-junctional filtering more efficient. Optogenetically-induced GABA release within DRG from the transplanted GABAergic cells enhanced filtering and reduced both acute and chronic inflammatory and neuropathic pain in vivo. These findings support 'gating' of pain information by spinal ganglia and suggest new therapeutic approaches for pain relief.
\end{abstract}




\section{INTRODUCTION}

Current understanding of the somatosensory information processing largely assumes that peripheral nerves faithfully deliver peripherally-born action potentials to the spinal cord. The first synapse in the dorsal horn of the spinal cord is assumed to be the first major integration point for action potentials generated at the periphery. Such a view is represented by the Gate Control Theory of pain (1) and its subsequent refinements and modifications (2-4). While it has been proposed that information processing is more efficient the earlier it begins within the sensory pathway (5-7), the absence of true synaptic connections or interneurons within peripheral somatosensory nerves and ganglia reasonably led researchers to dismiss them as possible information processing sites. Despite this, growing evidence suggests that a degree of crosstalk between the peripheral fibers $(8,9)$ or sensory neuron somata (10-12) might exist. Moreover, recent work suggests that action potentials propagating from the peripheral nerve endings of nociceptive nerve terminals to the spinal cord can fail (or be 'filtered') at axonal bifurcation points (t-junctions) within the spinal ganglia (13-19). An intrinsic GABAergic signalling system within spinal ganglia was recently proposed as a mechanism responsible for ganglionic filtering (15), yet, the understanding of how ascending information can be modified within spinal ganglia is sparse and direct evidence is missing. Here we obtained direct in vivo evidence for ganglionic filtering mediated by the GABA signalling system and assessed if such filtering can be exploited to control pain. Using in vivo electrophysiological recordings from the peripheral and central processes of the rodent sensory nerve, optogenetic manipulations, stem axon morphometry, and biophysical modelling we demonstrate that the spinal ganglion is a bona fide processing device controlling and modifying nociceptive signalling into the CNS. These findings support the existence of a 'peripheral gate' in somatosensory system and suggest new ways of how sensory ganglia can be targeted for pain control. Effective and safe pain relief is still an unresolved clinical problem with many mainstream CNS-targeting treatments being inadequate due to side effects, tolerance and addiction issues. Identification of the peripheral site of nociceptive information processing may shape safer and more efficient pain therapies.

\section{RESULTS}

Action potentials induced by the excitation of peripheral nerve endings are filtered within the DRG. We first developed a method for in vivo electrophysiological recording of extracellular spiking activity from both the peripheral and central branches of the L5 spinal nerve of a rat. Spinal nerve (SN), DRG, and dorsal root (DR) nerves were surgically exposed in anesthetized rat (Fig. 1A, B). SN and DR were then individually suspended on fine hook electrodes, while the DRG was exposed to direct drug application. This preparation allows $i$ ) synchronous measurement of the firing rates in the SN (before spikes enter the DRG) and DR (after spikes passed through the DRG); ii) sensory stimulation of the hind paw; iii) direct application of compounds or light to the DRG.

In our recordings both the SN and DR usually displayed spontaneous firing activity (Fig. 1C-H), consistent with earlier reports $(20,21)$. Intraplantar injection of algogenic compounds, capsaicin (a TRPV1 agonist; CAP, 10 $\mu \mathrm{M}, 50 \mu \mathrm{l}$; Fig. 1C, D) or bradykinin (BK, $100 \mu \mathrm{M}, 50 \mu \mathrm{l}$; Fig. 1E, F), significantly increased firing frequency in both $\mathrm{SN}$ and $\mathrm{DR}$ branches of the nerve, consistent with the evoked nociceptive inputs being transmitted from the peripheral nerve towards the spinal cord. Capsaicin injection increased firing rates to $288 \%$ and $295 \%$ of 
basal values in SN and DR nerves, respectively; BK injection increased firing rates in SN and DR to $166 \%$ and $246 \%$, respectively.

Recent studies suggest there is a GABAergic inhibition at the DRG, in addition to the well-accepted spinal GABAergic inhibitory network $(15,22,23)$. We thus tested how exogenous application of GABA to the DRG would affect the propagation of peripherally-induced nociceptive signals through the ganglion. Direct injection of GABA ( $200 \mu \mathrm{M}, 3 \mu \mathrm{l})$ into the DRG (see Methods) significantly reduced capsaicin- or BK-induced firing rates specifically in the DR, having no effect on the firing rates in the SN (Fig. 1C-F). Thus, GABAergic inhibition at the DRG can induce a prominent filtering of the throughput conduction.

Interestingly, application of the $\mathrm{GABA}_{\mathrm{A}}$ receptor antagonist, bicuculline (BIC; $200 \mu \mathrm{M}, 3 \mu \mathrm{l}$ ) to the DRG during continuous recording of tonic activity in both SN and DR (in the absence of any peripheral stimulation) significantly increased firing rate in the DR but not in the SN (Fig. 1G, H). This finding is consistent with our previous observation that application of BIC via the L5-DRG-implanted cannula in vivo induces nocifensive behavior towards the hind paw (15). Thus, BIC is likely to attenuate tonic filtering in nociceptive fibers passing through the DRG.

The interpretation of experiments presented in Fig. 1 could be complicated by the presence of the ventral root. Even though the experiments were conducted on immobile animals under deep anesthesia, the presence of intact motor fibers in the SN allows for execution of the flexor reflex which could contribute to SN activity via the efferent and re-afferent discharge. To account for such an eventuality, we evaluated the effect of ventral root (VR) transection on the spontaneous and evoked activity in SN and DR (Fig. S1A). VR transection had no noticeable effect on either the SN or DR spontaneous activity (Fig. S1B, C). Capsaicin, GABA (Fig. S1D, E) and BIC (Fig. S1F, G) produced effects qualitatively identical to these presented in Fig. 1: capsaicin increased firing rate in both $S N$ and $D R$ and GABA reduced this induced firing rate in DR but not SN. BIC increased firing rate in DR but not in SN. Interestingly, when GABA was DRG-injected on its own, without noxious stimulation of the paw, it failed to produce a significant effect on firing rate in both the SN and DR (Fig. $\mathrm{S} 1 \mathrm{H}, \mathrm{I})$. Another important observation from the experiments presented in Fig. 1 and Fig. $\mathrm{S} 1$ was that firing rates in the SN were consistently higher than in the DR. Importantly, this was also true for preparations with VR transection (summarized in Fig. S1J). Together with the fact that BIC consistently increased firing rates in the DR (with or without VR transection) these findings support the hypothesis for tonic filtering at DRG.

While VR transection eliminated the efferent input, it did not eliminate efferent fibers themselves from the spinal nerve, thus, any spurious activity in those fibers could have contributed to the SN activity and may have contributed to higher firing rates in the $\mathrm{SN}$, as compared to DR. In order to eliminate efferent fibers we took advantage of the fact that VR injury causes progressive degeneration of the motoneurons and preganglionic parasympathetic neurons (PPN) (24). Thus, we performed VR transections (Suppl. Methods) and allowed animals to recover; two weeks after VR transections the recordings similar to these shown in Fig. 1 and Fig. $\mathrm{S} 1$ were repeated. The motor and PPN fiber degeneration was confirmed by almost complete loss of their marker, Choline acetyltransferase (ChAT) (24) at two weeks after VR transection (Fig. S2A). Despite the removal of efferent fibers, tonic firing rate in the DR was still significantly lower, as compared to SN; GABA still significantly reduced the capsaicin-induced firing rate in DR but not $S N$, while BIC increased firing rate in DR but not in SN (Fig. S2B-E). Thus, under our experimental conditions, motor neuron input had no significant contribution to either spontaneous or to evoked activity in SN or DR. 
When we tested the effects of GABA on the capsaicin-induced firing and of BIC on spontaneous activity in SN and DR in female rats (Fig. S3) and on male rats anesthetized with different anesthetic (isoflurane instead of pentobarbital i.p.; Fig. S4), we obtained results qualitatively identical to these shown in Fig. 1 and Fig. S1-S2. Thus, GABA-mediated modulation of ganglionic filtering is a phenomenon reliably observed under a variety of experimental conditions in animals of either sex.

In order to better understand filtering of specific spikes in the recordings shown in Fig. 1 and Fig. S1 we developed a spike-matching method allowing to correlate SN and DR spikes (Fig. 2A; Fig. S5A). This method proved accurate $(80-100 \%)$ in Poisson-generated spike trains up to $100 \mathrm{~Hz}$ irrespective of the degree of filtering (not shown); the accuracy was inversely proportional to firing frequency (Fig. S5B). Spike sorting extracellular $\mathrm{SN}$ waveforms using the WaveClus implementation of super-paramagnetic clustering allowed us to isolate distinct spike clusters (units) and match these between SN and DR recordings. To rule out contamination of DR units with synchronized firing of another fiber, we calculated the mean deviation (represented as a z score) of each waveform in the unit from the mean waveform of the unit (Fig. S5C). Any spikes originating from another fiber firing in a temporally correlated way should exhibit a different waveform shape and thus be recognised as an outlier ( $>3 \mathrm{z}$ score). The large majority of spikes were within a $z$ score of 3 from the unit means, suggesting our matching protocol identified homogenous and distinct DR firing units.

A representative experiment capturing the firing activity of four distinct units is shown in Fig. 2B-D. Capsaicinevoked spike units were clearly identifiable, noted by the onset of activity during application of capsaicin (Fig. $2 \mathrm{C}, \mathrm{D}$ ); these were characterized by a larger amplitude on average (CAP-insensitive units: $14.5 \pm 1.5 \mu \mathrm{V}$; CAPsensitive units: $19.6 \pm 0.9 \mu \mathrm{V} ; \mathrm{p}<0.01)$. However, capsaicin-sensitive units could not be well distinguished by latency and spike width in this way. The mean drop in spiking units between SN and DR was significantly greater with GABA administration after CAP (Fig. 2E, F). To understand how features of the spike waveform predict spike failure when passing through the t-junction, we performed multiple linear regression analysis of spike width and amplitude. Spike amplitude was a significant predictor of filtering in the dorsal root with a coefficient of $1.908 \quad(p<0.01)$, indicating that larger amplitudes but not width corresponded with a greater probability of failure to propagate to the DR during baseline conditions. During administration of CAP, spike units with smaller widths (coefficient of $-23.35, p<0.001$ ) and a trend of larger amplitudes (coefficient of 1.29, $p=0.0825$ ) were more likely to be filtered. We further characterized spike units as either A-fibre or C-type based on conduction velocity $(<1.2 \mathrm{~m} / \mathrm{s}$; presumably C-type; $>1.2 \mathrm{~m} / \mathrm{s}$, presumably A-fibers (25)). Interestingly, this revealed that C-type fibres were indeed significantly more filtered during application of GABA (Fig. $2 \mathrm{G}$ ).

Taken together, data presented in Fig. 1, 2 and Suppl. Fig. 1-5 suggest: $i$ ) there is a basal activity in the SN and DR even in the absence of peripheral stimulation; ii) the firing rates in the SN are higher than these in DR, suggesting 'tonic filtering' of this basal activity at the DRG. iii) DRG-applied GABAA antagonist (BIC) increased basal firing in the DR but not in the $S N$, possibly suggesting removal of 'tonic' GABAergic inhibition in DRG. iv) Noxious stimulation increases firing rates in both $S N$ and $D R$, but the DRG-applied GABA reduced firing in the DR specifically, thus enhancing GABAergic filtering in the nociceptive fibers. Exogenous GABA did not affect the basal firing rate (either in the $\mathrm{SN}$ or $\mathrm{DR}$ ) indicating that there might be GABA-dependent and GABAindependent filtering mechanisms.

Transplantation of forebrain GABAergic neuron precursors into the adult mouse DRG in vivo delivers an analgesic mechanism. To test how GABAergic filtering of nociceptive transmission at the DRG can be exploited in vivo, we adopted an approach developed by Basbaum's group, who were able to transplant and 
bioRxiv preprint doi: https://doi.org/10.1101/2021.07.14.452325; this version posted October 20, 2021. The copyright holder for this preprint (which was not certified by peer review) is the author/funder. All rights reserved. No reuse allowed without permission.

functionally integrate into dorsal spinal cord, embryonic GABAergic progenitor cells from the medial ganglionic eminence (MGE). Transplanted MGE cells were able to compensate for the loss of spinal GABAergic inhibitory system observed in neuropathic pain models $(26,27)$. We transplanted embryonic MGE cells derived from VGAT-ChR2-eYFP into the L4 DRG of WT C57 mice. L4 DRG was chosen in this case as it is the major contributor to the sciatic nerve in mice (28). At four weeks after the DRG injection we observed numerous YFP-positive cells in the DRG sections (Fig. 3A); fluorescent cells were entirely absent in vehicle-injected control animals.

In order to confirm that transplanted MGE cells can function as GABAergic neurons within DRG, we performed patch-clamp recordings from the DRG neurons juxtaposed to the MGE cells (Fig. 3B-D) using 'loosened' whole L4 DRGs from mice pre-injected (4 weeks) with the VGAT-ChR2-eYFP-expressing MGE (see Methods). Stimulation of the ganglion with the $473 \mathrm{~nm}$ blue light induced inward currents in 9/9 DRG neurons, which were in close juxtaposition with MGE cells (Fig. 3B-D). These same neurons also responded to perfusion of $200 \mu \mathrm{M}$ GABA with very similar inward currents. In contrast, DRG neurons from vehicle-injected mice never responded to blue light (0/8 neurons) but these did respond to GABA (Fig. 3C, D). These results suggest that $i$ ) implanted MGE progenitor cells can survive and maturate to produce GABA-releasing neurons in DRG; ii) stimulusinduced release of GABA by resident neurons can induce a response in neighboring neurons.

Next, we tested if optogenetic release of GABA from the implanted MGE cells can alleviate chronic pain. In these experiments a fiber-optic light guide was implanted into the DRG immediately after the MGE cells transplantation (Fig. 3E; Methods). Chronic inflammation with hind paw injection of complete Freund's adjuvant (CFA, $20 \mu \mathrm{l}$ ) induced significant mechanical and thermal hyperalgesia (Fig. 3F-H). We then performed mechanical (Fig. 3G) or thermal (Fig. 3H) sensitivity tests while stimulating ipsilateral L4 DRG with blue light. Optical stimulation significantly reduced both types of hyperalgesia in MGE-injected mice. Interestingly, starting from the second week after the CFA injection, both mechanical and thermal hyperalgesia in the MGEimplanted mice started to recover even in the absence of optogenetic stimulation and by the $3^{\text {rd }}$ week after the CFA injection blue light stimulation no longer produced any additional analgesic effect (Fig. 3G, H). We hypothesized that a buildup of tonic GABA release from the transplanted MGE cells in DRG could be responsible for the light-stimulation-independent recovery of the CFA-induced hyperalgesia. This hypothesis was corroborated in experiments, similar to the ones presented in Fig. 3G, H, but in which no optogenetic stimulation was used, to avoid inducing any stimulus-induced GABA release (Fig. S6A, B). We also utilized a chronic constriction injury $(\mathrm{CCl})$ model of neuropathic pain in similar experiments (Fig. S6C, D). In both models hyperalgesia developed in control (vehicle-injected) and MGE-implanted mice. However, the latter group displayed significantly quicker and more complete recovery. Collectively, these data suggest that DRGimplanted MGE cells can be stimulated to release GABA locally in vivo and that such release produces analgesic effect.

\section{Optogenetic release or direct injection of GABA to the DRG enhances filtering of spikes triggered by} noxious but not innocuous stimuli. We hypothesized that the analgesic effect of MGE cells transplanted into the DRG is mediated by GABAergic filtering of pro-nociceptive spikes at the DRG. To test this we used an approach similar to that used in Fig. 1 and Suppl. Fig 1-5, but instead of applying GABA, we stimulated L4 DRG with blue laser light (Fig. 4A). Optogenetic DRG stimulation (3 - 4 weeks after MGE transplantation) gave rise to qualitatively very similar effects to these produced by application of GABA. Firing induced by the hind paw injections of capsaicin $(10 \mu \mathrm{M}, 20 \mu \mathrm{l}$, Fig. 4B, D) or BK (100 $\mu \mathrm{M}, 20 \mu \mathrm{l}$, Fig. 4C, E) was significantly 
inhibited by the light stimulation in DR but not SN. The optogenetic suppression of firing in DR was evident immediately upon application of blue light (Fig. 4B, C; lower right panels).

Application of noxious heat ( $60^{\circ} \mathrm{C}$ water; Fig. S7A, B) and noxious cold (ice; Fig. S7C, D) induced a significant increase of firing frequencies in both $S N$ and $D R$ and optogenetic DRG stimulation significantly inhibited firing rates in DR but not in SN. We then tested innocuous and noxious mechanical stimulation. Air puffs and subthreshold (4g) von Frey filament stimulation (Fig. S7E-H) increased firing in both SN and DR, presumably via the activation of low threshold mechanoreceptors in the skin $(29,30)$. Interestingly, optogenetic stimulation of DRGs did not significantly affect the firing frequency in either SN or DR in these experiments (Fig. S7E-H). Noxious mechanical stimulation of the paw with a blunt glass needle also significantly increased firing frequencies in both $S N$ and DR. In this case optogenetic stimulation substantially inhibited firing in DR, but not in SN (Fig. S7I, J). Thus, it appears that GABAergic filtering in DRG predominantly exists in nociceptive fibers.

Next, we performed a set of experiments, similar to that shown in Fig. S7 but in naïve rats and with the direct injection of GABA into the DRG instead of optogenetic stimulation (Fig. S8). There was a pattern similar to that observed with optogenetic stimulation of MGE cells in mice. Firing induced by noxious heat (Fig. S8A, B) and noxious cold (Fig. S8C, D) was selectively inhibited in the DR but not in the SN by the DRG-applied GABA. No significant effects of DRG-applied GABA were seen when firing was induced by innocuous air puffs or subthreshold von Frey hairs (Fig. S8E-H). In contrast, firing induced by the noxious needle prick was reduced in the DR but not in the SN (Fig. S8I, J). Striking similarity of the effects of exogenous GABA and optogenetic GABA release in DRG strongly suggest that i) the GABAergic system controls filtering efficacy of the ganglia; ii) the filtering is most efficient in nociceptive fibers and iii) such filtering is the most plausible explanation of the analgesic effect of MGE cells transplanted to the DRG; iv) GABAergic filtering exists in both rats and mice.

To test this further we performed single-unit recordings (Fig. 5A); firing was induced by a stimulating electrode placed in SN and recordings were made from the mechanically isolated teased DR bundles. A-type and Ctype spikes were distinguished by the conduction velocity (A fibers $>1.2 \mathrm{~m} / \mathrm{s} ; C$ fibers $<1.2 \mathrm{~m} / \mathrm{s}(25)$ ). The firing in both fiber types was blocked by $1 \mu \mathrm{M}$ TTX applied to the DRG (Fig. S9). Evoked stimuli propagated reliably in both fiber types under control conditions with only occasional failures (basal failure rate in $A$ fibers: $6.3 \pm 2.6 \%$, $n=8$; C fibers: $16.3 \pm 2.6 \%, n=8$; Fig. $5 B, C$ ), which is consistent with earlier report (18). DRG-applied GABA significantly increased failure rate in $C$ fibers (to $63.8 \pm 3.24 \%, p<0.001$ ) but had no significant effect in $A$ fibers (Fig. 5B, C). Due to technical difficulties of these recordings not all of these were long enough to reliably analyze failure rate but amongst all the recordings made, $89 \%$ (34/38) of $C$ fibers and only $24 \%(11 / 45)$ of $A$ type fibers displayed GABA-induced t-junctional spike failure, while in $100 \%$ of both fiber types failure was induced by TTX (Fig. 5D, Fig. S9). Taken together, single-unit recordings revealed that DRG application of GABA selectively increases filtering of C-fiber activity.

Soma has more influence over t-junctional filtering in C-type as compared with A-type fibers. DRG neurons are pseudo-unipolar and axonal bifurcation (t-junction) is potentially a major site of spike filtering in DRG due to impedance mismatch $(13,15-17,31-33)$. Why is GABA-induced filtering more efficient in the Cfibers, as compared to A-fibers? One possibility is the different length of the stem axon (from the soma to the $\mathrm{t}$-junction) and, thus, the electrotonic influence of the soma on the t-junction and spike propagation: the longer the stem axon, the poorer the coupling $(14,15,17)$. To our knowledge, there has been no systematic analysis of stem axon lengths in mammalian DRG neurons, although drawings by Ramon y Cajal depict much shorter 
stems in small-diameter neurons, as compared to larger ones (34). In addition, larger neurons are often displayed having stems with a winding 'glomerular' section, extending the length (34-36). In order to assess stem length in C-type vs. A-type fibers we cleared (37) rat whole DRG mounts and immunostained them with the C-fiber marker, peripherin, and A-fiber marker, neurofilament-200 (NF-200). We then performed light-sheet microscopy of entire ganglia (Movie S1) and measured the stem axon lengths of peripherin-positive and NF200 positive neurons (Movie S2; Fig. 6A-B). Consistently, peripherin labelled neurons with much smaller somatic diameter, as compared to NF-200 positive neurons $(26.1 \pm 0.4 \mu \mathrm{m} v s .42 .4 \pm 0.8 \mu \mathrm{m}, \mathrm{p}<0.001$; Fig. $6 \mathrm{~A}$, E; Fig. S10). Stem axon diameters of peripherin-labelled neurons were also consistently smaller $(1.34 \pm 0.03$ $\mu \mathrm{m}$ vs. $2.1 \pm 0.1 \mu \mathrm{m}$, p<0.001; Fig. 6D; Suppl. Fig. 10). Peripherin labelled neurons displayed much shorter stems, as compared to NF-200 positive neurons $(60.7 \pm 4.2 \mu \mathrm{m}$ vs. $232.5 \pm 22.9 \mu \mathrm{m}, \mathrm{p}<0.001$; Fig. $6 \mathrm{C}$; Suppl. Fig. 10). While for all peripherin-positive neurons analyzed in Fig. $6 \mathrm{C}$ and Suppl. Fig. 10, the t-junction was reliably identified (Movie S2), it was often impossible to confidently locate the t-junctions of NF-200 positive neurons as these were too far away from the cell body. In these instances stem length was recorded as the longest traceable distance and, hence, it is an underestimation of the real stem length.

We hypothesized that GABAergic control of DRG filtering is less effective in A-fibers compared to C-fibers due to differences in stem axon length. Our previous computational modeling suggested that in a C-fiber with 75 $\mu \mathrm{m}$ stem axon, activation of somatic GABAA channels can indeed cause a failure of action potential to propagate through the t-junction due to a combination of the impedance drop and sodium channel inactivation (15). But even though data presented in Fig. $6 \mathrm{~A}-\mathrm{C}$ indicate that the stem axon of $\mathrm{C}$-fibers is, on average, at least 3 times shorter than that of A-fibers, myelination could possibly compensate for the longer distance. Using a computational model of an A-fiber neuron, we examined the relationship between somatic conductance and stem axon length. We constructed a minimal model of an A-type neuron (see Suppl. Methods) containing a limited repertoire of voltage-gated channels and a geometry consistent with the parameters obtained from the light-sheet morphometry of the NF-200 positive neurons and compared it to a model with a C-fiber morphology (15).

We first examined the influence of changes in membrane potential at the soma on the t-junction. We calculated the voltage transfer ratio, the fractional potential reaching the $t$-junction by a potential generated at the soma, as a function of stem axon length (Fig. $6 \mathrm{~F}$ ). In C-fibers, the transfer ratio was greater than 0.75 for a physiological range of stem lengths, while it was less than 0.6 for stem axon lengths measured from A-type neurons. For A-fibers, similar results were obtained by either adjusting the number of internode/node pairs or internode distance. Thus, the modeling predicts that the soma of a $\mathrm{C}$-fiber neuron should have more influence on the t-junction than an A-type neuron soma.

In A-fiber with stem axon of $400 \mu \mathrm{m}$, propagating spikes at the nodes of Ranvier were $78-102 \mathrm{mV}$ in amplitude $\left(\mathrm{G}_{\mathrm{Na}}=0.5-1 \mathrm{mS} / \mathrm{cm}^{2}\right)$ and had conduction velocities of $11 \mathrm{~m} / \mathrm{s}$ for $\mathrm{G}_{\mathrm{Na}} \geq 1 \mathrm{~S} / \mathrm{cm}^{2}$. In a C-fiber with a stem axon of $75 \mu \mathrm{m}$, propagating spikes were $94-101 \mathrm{mV}$ in amplitude with a maximum conduction velocity of $0.4 \mathrm{~m} / \mathrm{s}$ (Fig. 6G, left). Through-conduction across a wide range of physiologically-relevant parameter space was not influenced by firing at the soma, consistent with the earlier model (38). To assess the potential effect of GABA receptor activation on spike propagation through the $D R G$, we introduced a chloride conductance $\left(\mathrm{G}_{\mathrm{CI}}\right)$ in the soma that depolarized the t-junction and reduced local spike amplitude (Fig. $6 \mathrm{G}$, right). The threshold $\mathrm{G}_{\mathrm{Cl}}$ for blocking spike propagation in the $\mathrm{C}$-fiber model was approximately 10 -fold lower than for the A-fiber model. 
We next systematically examined the relationship between somatic $\mathrm{G}_{\mathrm{Cl}}$ and $\mathrm{G}_{\mathrm{Na}}$ (Fig. $6 \mathrm{H}$ ) and found that spike propagation in this A-fiber model could only be blocked when $\mathrm{G}_{\mathrm{Na}}$ was less than $0.8 \mathrm{mS} / \mathrm{cm}^{2}$ and the minimum chloride conductance needed was $\sim 10$-fold higher than for the C-fiber model, which was sensitive up to a $\mathrm{G}_{\mathrm{Na}}$ of $1 \mathrm{mS} / \mathrm{cm}^{2}$. As expected, when the stem axon length of either model was systematically varied, we found that longer stem axons limited the influence of soma conductance on spike propagation (not shown). Taken together, the morphometry of DRG neuron axons and our biophysical model supports the hypothesis that the longer stem axons of A-fibers limit the influence of somatic GABA $A_{A}$ conductances on spike filtering.

Evidence for tonic release of GABA within the DRG. Our paired recordings from SN and DR suggest the existence of tonic filtering at the DRG. There appears to be at least two different types of tonic filtering: in nociceptive fibers it appears to be GABAergic since BIC increases firing rate in the DR (Fig. 1G, H; Fig. S1S4) and, when injected to the DRG in vivo, induces pain-like behavior (15)). In non-nociceptive fibers it is likely to be mediated by a different mechanism since i) poor electrotonic coupling between soma and t-junction (Fig. 6) and ii) lack of effect of DRG-applied GABA on basal firing rate in the DR (Fig. S1H, I). Here we focused on the GABAergic mechanism; our previous data suggested that some DRG neuron cell bodies are capable of releasing GABA upon stimulation (15). Another recent study reported robust activity-dependent somatic vesicle release from DRG neurons (39). To investigate mechanisms of GABA release by DRG neurons we developed a method for measuring exocytosis of GABA-containing vesicles based on the live uptake of luminal (C-terminal) antibody against vesicular GABA transporter VGAT (Fig. 7A). N-terminus of VGAT, inserted in the neurotransmitter vesicle membrane, faces the cytoplasm while the $C$ terminus resides in the vesicle lumen (40). During exocytosis and subsequent recycling of a vesicle, luminal VGAT epitopes are temporarily exposed to the extracellular milieu. During such an exposure, antibodies that recognize these epitopes can bind to these and become trapped and subsequently internalized by endocytosis (Fig. 7A; (40)). Antibodies against the Nterminus of VGAT should not be entrapped in this way as N-terminus of VGAT remains cytosolic at all times. Depolarization of cultured DRG neurons with extracellular solution containing $100 \mathrm{mM} \mathrm{KCl}$ induced robust uptake of C-terminal (luminal) but not N-terminal (cytosolic) VGAT antibody by DRG neurons (Fig. 7B, C; quantified as proportions of neurons stained with the C-terminal VGAT antibody). The depolarization-induced C-terminal VGAT antibody uptake was significantly reduced (but not abolished - in good agreement with (39)) by the removal of extracellular $\mathrm{Ca}^{2+}$ (Fig. 7B, C). Interestingly, even in the absence of depolarization, there was a significant number of neurons that took up C-terminal VGAT antibody (Fig. 7B top panel; C), hinting at spontaneous exocytosis of VGAT-containing vesicles. The N-terminal VGAT antibody was not taken up by DRG neurons (Fig. 7B, bottom panel; C), even though this same antibody labeled permeabelized DRG neurons well (15).

Additionally, we used live imaging to optically monitor GABA release from the DRG neurons in culture. We transfected HEK293 cells with $\alpha_{1}, \beta_{2}$, and $\gamma_{2}$ GABA $A_{A}$ subunits and a halide-sensitive EYFP mutant (H148Q/I152L; EYFP-QL). The fluorescence of EYFP-QL is quenched by iodide and since $\mathrm{Cl}^{-}$channels (e.g. $G_{A B A_{A}}$ ) are permeable to this anion, EYFP-QL fluorescence quenching by $\mathrm{I}^{-}$can be used to monitor $\mathrm{Cl}^{-}$ channel activation (41-44). We co-cultured these 'GABA indicator' HEK293 cells with DRG neurons and measured EYFP-QL fluorescence quenching induced by the addition of $5 \mathrm{mM} \mathrm{Nal}$ to the extracellular solution (Fig. 7D). Nal induced robust EYFP-QL fluorescence quenching only when the indicator cells were co-cultured with DRG, not in the monoculture (Fig. 7E). Moreover, this quenching in the DRG/indicator cell co-culture was significantly reduced in the presence of $50 \mu \mathrm{M} \mathrm{BIC} \mathrm{(Fig.} \mathrm{7E,} \mathrm{F).} \mathrm{BIC} \mathrm{also} \mathrm{significantly} \mathrm{slowed} \mathrm{down} \mathrm{the} \mathrm{kinetics}$ 
of the EYFP-QL fluorescence intensity decay (Fig. 7G). These data further support existence of GABA tone in DRG.

\section{DISCUSSION}

There is substantial evidence indicating that spinal ganglia regulate the flow of sensory information through to the CNS and that this processing can be modulated by GABAergic signalling. Thus, it was shown that DRG neurons can produce and release inhibitory neurotransmitter $\operatorname{GABA}(15,23)$, GABA delivered specifically into the DRG has prominent analgesic action $(15,22)$ and that electrical stimulation of DRGs provides strong analgesia even to the most intractable types of chronic pain in humans (45-47). Experimental measurements $(13,14,18,19,48-50)$ and simulations $(14-17,51)$ suggested axonal t-junctions as biophysically amenable sites for peripheral filtering/gating and modulation by intrinsic GABA signalling.

Our main findings here are threefold. First, we demonstrate that the DRG exerts a dynamic control over the throughput conduction via GABA receptor activation. Indeed, focal application of GABA, or optogenetic stimulation of GABA release from the DRG-transplanted MGE cells, specifically reduced stimulus-induced firing frequencies in the DR but not the $S N$ in various experimental settings. Second, we show that this GABAergic filtering is much more efficient in the C-fibers, as compared to A-fibers. Third, we demonstrate that an intrinsic GABAergic inhibitory system can be engaged to scale the filtering of spikes passing through the DRG up or down, modulating nociceptive input to the CNS. Importantly, we further show that this mechanism can provide effective relief of both acute and chronic pain.

The t-junctions, where the stem axon bifurcates into SN and DR axons, are expected to have a lowered safety factor for AP propagation due to the impedance mismatch $(13-15,32,49,52)$. Our earlier biophysical model of a $\mathrm{C}$-fiber predicted that opening of somatic/peri-somatic $G A B A_{A}$ channels promotes failure of action potential propagation through the t-junction due to a combination of input impedance drop, GABA conductance shunting, and voltage-gated sodium channel inactivation (15). Here we provide strong experimental support to these predictions. We show that GABA receptor activation within the DRG reduces the evoked firing rate recorded at a point immediately after the t-junction, and has no effect on spikes entering the DRG. The most straightforward interpretation of these observations is that evoked spikes in peripheral axons fail to propagate through the DRG into DR axons when GABA system is activated in the DRG.

Another striking finding is that DRG filtering is much more robust in C-, as compared to the A-fibers. As a consequence, optogenetic GABA release (Fig. S7) or DRG injection of GABA (Fig. S8) specifically inhibited firing produced by noxious but not innocuous stimuli. We propose that the induced filtering is more efficient in the $\mathrm{C}$-fibers because these have a shorter stem axon. Electrotonic coupling of the soma to the t-junction depends on the stem axon length $(15,17)$. Drawings by Ramon y Cajal indeed suggested much shorter stems in small-diameter DRG neurons, as compared to the larger ones (34). Our light-sheet imaging of cleared DRG with labelled C- and A-type fibers established that stem axon is indeed over 3 times shorter in C fibers (Fig. 6). Our new biophysical model of an A-fiber suggests that when a stem axon is longer than 200-300 $\mu \mathrm{m}$, opening of somatic $\mathrm{GABA}_{A}$ channels has minimal effect on the action potentials propagating through their $\mathrm{t}$ junctions. Thus, this investigation may have revealed a simple but elegant principle of differential filtering of spikes that depends on the modality-specific fiber morphology. It has to be acknowledged that other 
mechanisms, e.g. differential ion channel densities (including but not limited to $G A B A_{A}$ ) at the t-junctions or stem axon of different fiber types could also contribute to the differential filtering.

Interestingly, a recent study used single unit recordings from the DR to demonstrate that electric field stimulation of the DRG in rats, which mimicked DRG field stimulation (neuromodulation) used for analgesia in humans (45-47), increased t-junctional filtering mostly in C-fibers, with little effect in A-type fibers (19). Our results are in good agreement with these findings; together these data could explain otherwise 'paradoxical' (but effective) analgesic approach via electrical DRG stimulation.

Two consistent, but yet to be fully understood, observations reported here require further consideration. First, there was considerable basal activity under control (no peripheral stimulation) conditions. This was unexpected and may arise from the preparation-specific injury. However, our previous observation that DRG-injected GABA antagonists in freely behaving animals induced pain-like paw flinching in the absence of noxious input (15) may suggest that there is indeed some tonic activity in the peripheral nerve which is failing to reach spinal cord. Second, basal firing rates in the DR were almost invariantly about $50 \%$ lower, as compared to these in SN, even under conditions where efferent fibers were either cut (Fig. S1) or physically destroyed (Fig. S2). Single-unit recordings (Fig. 5) revealed that evoked spikes in $\mathrm{C}$ and $\mathrm{A}$ fibers do fail occasionally under basal conditions (at a rate of $\sim 16 \%$ in $C$ and $\sim 6 \%$ in $A$ fibers), which was consistent with a recent report (18). Thus, basal filtering does exist but when measuring evoked single-unit spikes it is much lower than $\sim 50 \%$ mismatch seen in our multi-unit SN/DR recordings. While $G_{A B A}$ antagonist BIC significantly reduced this mismatch indicating that at least part of it is due to tonic GABAergic inhibition, we cannot exclude that other factors may contribute to the mismatch, including e.g. some back-propagation in the SN. It is important to note however that regardless of the nature of this mismatch in basal firing, the sensory stimulation of the paw (noxious or innocuous) is seen in our SN/DR recordings as a consistent increase in the firing rates in both compartments ( $\mathrm{SN}$ and DR). Moreover, we were able to identify individual capsaicin-induced units using our spike matching algorithm (Fig. 2, Fig. S5). Thus, the activity induced by noxious stimulation is reliably detectable on the background of the basal activity. Importantly, it is this induced noxious activity, which is specifically filtered at the DRG by the GABAergic system.

Another important question - is the intrinsic GABAergic system in the DRG sufficient to impose filtering in vivo? Strong expression of functional GABA receptors in DRG is well recognized (reviewed in (53)) but the ability of DRG to produce and release GABA is less well documented (although see $(15,23)$. Transcript levels of enzymes (GAD65, GAD67) and transporters (VGAT) needed to synthesize and package GABA into vesicles are low according to transcriptomic studies $(54,55)$. Yet functional proteins are detectable $(15,23,56)$, as is tonic and induced GABA release (Fig. 7 and (15)). It has to be pointed that a non-vesicular mechanism for GABA release that does not require VGAT also exists (i.e. via LRRC8 channels (57)). Another important evidence for endogenous GABA tone is the in vivo studies that demonstrated that GABA reuptake inhibitor, NO711, produced analgesic effect when delivered to the DRG in vivo $(15,22)$. DRG-applied GABAA antagonists, on the other hand, exacerbated peripherally-induced pain and even produced a pain-like behavior (15). Hence, there must be sufficient amount of endogenous GABA in the DRG to control the 'filter'.

A major causative factor of neuropathic pain is a loss of dorsal horn GABAergic inhibitory system (58). GABAergic progenitor cells implanted into adult spinal cord survive and integrate into the spinal inhibitory circuit $(27,59,60)$, reducing neuropathic pain severity $(26,61)$. We found the MGE cells transplanted into the DRG of adult mice also survive there and can release GABA (Fig. 3). Optogenetic stimulation of MGE- 
transplanted DRG significantly alleviated chronic inflammatory hyperalgesia (Fig. 3). Importantly, even without optogenetic stimulation, MGE cell transplantation accelerated the recovery from both inflammatory and neuropathic types of hyperalgesia (Fig. S6). This, together with observed higher efficiency of GABAergic filtering at the $C$, as compared to $A$ fibers might have a therapeutic significance as it suggests that GABAergic system in DRG could be targeted for pain relief without significantly compromising other haptic sensations. In the spinal cord MGE cells maturate into interneurons and integrate into the existing spinal inhibitory system $(26,27)$. For the case of the DRG transplant, the direct analogy is unlikely as there are no 'classical' interneurons in DRG. The most straightforward explanation for the anti-nociceptive effect of MGE cells in DRG is a 'GABA pump' mechanism, whereby MGE cells simply release GABA into the extracellular space thus increasing the GABA tone. Recent years saw increasing success in using stem cells as a chronic pain therapy $(62,63)$ and GABAergic progenitor cells can be generated from human stem cells $(64)$ and integrated into the pain pathways (65). Thus, targeting GABAergic system with the DRG-directed stem cell therapy or GABAmimetics tailored to reduce their CNS permeability could open up avenues for analgesic strategies with reduced CNS side effects.

\section{MATERIALS AND METHODS}

All animal experiments were performed with the approval of the Animal Care and Ethics Committee of Hebei Medical University under the International Association for the Study of Pain guidelines.

In vivo recording of peripheral nerve activity. All surgical procedures were performed under deep anesthesia with an i.p. injection of pentobarbital sodium (60-80 mg/kg) in accordance with the Animal Care and Ethical Committee of Hebei Medical University under the International Association for the Study of Pain guidelines. In one set of experiments (Fig. S4) pentobarbital was replaced by isoflurane ( $4 \%$ for induction, $2 \%$ for maintenance). Laminectomy was performed to expose right L5 DRG of adult male rat (Sprague-Dawley, 180-200 g) or L4 DRG of adult male C57BL/6J mice. Dorsal root (DR), spinal nerve (SN) and DRG were exposed by removal of both spinous and transverse processes of the vertebra bone; the DR and SN were then suspended on the hooked stainless-steel recording electrodes connected to BL-420F biological data acquisition and analysis system (Chengdu Techman Software Co., Ltd. China). The wound was filled with paraffin in order to stabilize preparation. The right hindpaw was used for the injection of capsaicin (10 $\mu \mathrm{M} ; 50$ $\mu \mathrm{l}$ for rat; $20 \mu \mathrm{l}$ for mouse) or Bradykinin (100 $\mu \mathrm{M}, 50 \mu \mathrm{l}$ for rat; $20 \mu \mathrm{l}$ for mouse), or the stimulation with hot water $\left(\sim 60^{\circ} \mathrm{C}\right)$, ice, air puffs (using aurilave), von Frey filaments ( $4 \mathrm{~g}$ for rat; $0.4 \mathrm{~g}$ for mouse) or needle prick (glass electrode). GABA (200 $\mu \mathrm{M} ; 3 \mu \mathrm{l}$ for rat; $2 \mu \mathrm{l}$ for mouse) or Bicuculline ( $200 \mu \mathrm{M} ; 3 \mu \mathrm{l}$ for rat; $2 \mu \mathrm{l}$ for mouse) was accurately delivered to the surface of exposed DRG by micropipettor penetrating through paraffin.

For the single unit recording in rats, dorsal root and spinal nerve were exposed and covered with liquid paraffin. A single axon bundle was teased away from dorsal root by a fine tweezer and placed on nerve fiber electrode for electrophysiological recording. The stimulus current delivered to the spinal nerve was gradually raised (10 $\mathrm{Hz}, 2$ - $5 \mathrm{~mA}$ ). Conduction velocity of fiber was determined by dividing conduction distance by response latency to electrical stimulus. GABA $(200 \mu \mathrm{M}, 3 \mu \mathrm{l})$ or Tetrodotoxin (TTX, $1 \mu \mathrm{M}, 3 \mu \mathrm{l})$ was accurately delivered to DRG by micropipettor.

Spike sorting. Electrophysiological recordings of both the spinal nerve and dorsal root were imported into Python and high pass filtered at $60 \mathrm{~Hz}$ using a digital Butterworth filter from the Scipy module (66). Extracellular 
spike times and waveforms were extracted using an absolute median deviation of between 5 and 6 from the median of the signal. Extracted spike waveforms were sorted using the WaveClus program (67) in Matlab to define individual neuronal units underlying the extracellular signal. Matching of spikes in the dorsal root to an origin spike in the spinal nerve was achieved by finding the minimum latency (within a tolerance window of the slowest theoretical fibre conduction velocity of $0.1 \mathrm{~m} / \mathrm{s}$ ) between spikes in the spinal nerve and dorsal root. Propagation failure was defined when a spinal nerve spike did not have a matching dorsal root spike within the tolerance window.

MGE cells transplantation. The female VGAT-ChR2-EYFP mice (Jackson Laboratory) with embryos between E12.5 and E13.5 were anesthetized with an intraperitoneal injection of pentobarbital sodium (60-80 $\mathrm{mg} / \mathrm{kg}$ ). All the embryos were removed via abdominal incision and placed into a $10 \mathrm{~cm}$ Petri dish containing ice-cold HBSS. The mice were humanely sacrificed. The gestational sac of each embryo was removed using forceps under the stereo microscope (SZX7, Olympus). The embryonic brain was extracted and cut along the sagittal plane to separate two hemispheres. Medial ganglionic eminence (MGE) on each hemisphere was then removed with a scalpel. MGE tissue was put in a $1.5 \mathrm{ml}$ collection tube containing $500 \mu \mathrm{l}$ DMEM/10\% FBS (Sigma) and triturated into a single cell suspension as described(68). The final cell density in the suspension of embryonic stem cells was measured with hemocytometer. Adult (5-6 weeks) male C57BL/6J mice were anesthetized with an intraperitoneal injection of pentobarbital sodium (60 - $80 \mathrm{mg} / \mathrm{kg})$. L4 DRG was exposed by removal of both spinous and transverse processes of the vertebra bone. The microinjector (Hamilton Co.) loaded with a suspension of MGE cells $\left(3 \mu \mathrm{l} ; \sim 1 \times 10^{7} / \mathrm{ml}\right)$ was inserted into the ganglion to a depth of $200 \mu \mathrm{m}$ from the exposed surface. The cell suspension was injected slowly, and the needle was removed 3 minutes after the injection. The muscles overlying the spinal cord were loosely sutured together, and the wound was closed. Animals developing signs of distress were humanely sacrificed. In order to verify that the DRGs were transplanted with MGE cells successfully, the L4 DRGs were excised, submerged in Tissue-Tek O.C.T. (Sakura, Alphen aan den Rijn, The Netherlands), frozen, and sectioned (10 $\mu \mathrm{m})$ using a freezing microtome (CM1950, Leica Microsystems). Slices were then analyzed for the EYFP fluorescence using confocal microscopy (TCS SP5 II, Leica Microsystems).

Chronic pain models and Behavioral tests. Chronic constriction injury $(\mathrm{CCl})$ and mechanical and thermal sensitivity tests were performed as described previously (15), further details are provided in Supplementary Methods.

In vivo optogenetic stimulation. Adult male C57BL/6J mice were $L 4 D R G$ transplanted with MGE cells from VGAT-ChR2-EYFP mice 3-4 weeks before experiments. Recordings of DR and SN activity was performed as described above in combination with laser stimulation $(473 \mathrm{~nm}, 3 \mathrm{~mW}, 30 \mathrm{~Hz}$ for 10 seconds with 20-second interval) of DRG using an MLL-FN-473-50 unit (Changchun New Industries Optoelectronics Technology Co., Ltd.) controlled by a pulsing set (S48 Stimulator, Grass Technologies, An Astro-Med, Inc. Product Group). In the behavioral tests on freely moving animals, a stainless steel cannula guide (RWD Life Science Co. Ltd., China; diameter $0.64 \mathrm{~mm}$ ) was implanted inot the L4 DRG, the cannula was firmly fixed in place with dental cement, and the optical fiber (RWD Life Science Co. Ltd., China; diameter $0.2 \mathrm{~mm}$, length $1 \mathrm{~m}$ ) was inserted through the guide; a more detailed description is provided in (15).

Patch clamp recording from DRG neurons. DRG dissection and recording were performed as described previously (69), further details are provided in Supplementary Methods. 
VGAT antibody uptake. DRG neurons were dissociated and cultured as described previously(69). DRG neurons were incubated for $15 \mathrm{~min}$ in either normal or 'high $\mathrm{K}^{+\prime}$ extracellular $(E C)$ solution supplemented with either C-terminal (luminal) N-terminal (cytosolic) VGAT antibodies. Normal EC solution contains (in mM): 144 $\mathrm{NaCl}, 5.8 \mathrm{KCl}, 1.3 \mathrm{CaCl}_{2}, 5.6 \mathrm{D}$-glucose, $0.7 \mathrm{NaH}_{2} \mathrm{PO} 4,0.9 \mathrm{MgCl}_{2}$ and $10 \mathrm{HEPES}$ (all from Sigma). In high $\mathrm{K}^{+}$ $\mathrm{EC}$ solution $\mathrm{NaCl}$ concentration was lowered to $49.8 \mathrm{mM}$ and $\mathrm{KCl}$ concentration was raised to $100 \mathrm{mM}$. $\mathrm{Ca}^{2+}$ free $\mathrm{EC}$ solution was also used; in this solution $\mathrm{CaCl}_{2}$ was omitted. After incubation cell cultures were washed 3 times with PBS and fixed using $4 \%$ paraformaldehyde, followed by permeabilization with $0.05 \%$ tween 20 and $0.25 \%$ triton-X 100 (with donkey serum) for $1 \mathrm{hr}$. Cells were then labelled with secondary antibody, washed three times with PBS, mounted on coverslips and imaged using Zeiss LSM880 confocal microscope. The following antibodies were used: VGAT C-terminal antibody (rabbit polyclonal \#AB-N44, Advance Targeting System; 1:200); VGAT N-terminal antibody (rabbit polyclonal 131002, Invitrogen, Eugene, Oregon, USA; 1:1000); secondary antibody: alexafluor donkey anti-rabbit 488 (Invitrogen, Eugene, Oregon, USA; 1:1000).

lodide imaging. HEK293 cells were co-transfected with cDNA encoding human $\alpha 1, \beta 2$ and $\gamma^{2}$ subunits of GABA $_{A}$ receptors (gift of David Weiss, Department of Physiology, University of Texas Health Science Center, San Antonio, Texas, USA) together with the halide-sensitive EYFP mutant (H148Q/I152L; EYFP-QL) using FuGENE® HD transfection reagent. Transfected cells were co-cultured for 24 hrs with DRG neurons isolated as described above (see also (15)). Extracellular solution consisted of (mM): $\mathrm{NaCl}$ (160); $\mathrm{KCl}(2.5) ; \mathrm{MgCl}_{2}$ (1); $\mathrm{CaCl}_{2}$ (2); HEPES (10) and Glucose (10); $\mathrm{pH}$ adjusted to 7.4 with $\mathrm{NaOH}$ (All from Sigma). I- containing solution was produced by equimolar substitution of $5 \mathrm{mM} \mathrm{NaCl}$ with Nal. I- imaging was performed using Nikon TE2000 E Swept Field Confocal microscope using 488nm argon laser as excitation light source. Images were recorded and analyzed using Nikon Elements software.

Dorsal root ganglia clearing, staining and morphometry. DRGs tissue clearing was performed using the iDISCO+ protocol (37). Cleared DRG samples were imaged using Ultramicroscope II (LaVision BioTech) Images were analyzed using the simple neurite tracer in FIJI. Using this semi-automatic method of tracing (Movie S2), stem axons were measured from origin at peripherin and NF-200-positive somata through the image stack until clear bifurcation or in the case of NF-200-positive cells, loss of a clear signal. Further details are provided in Supplementary Methods.

Computer modelling. All simulations were performed using NEURON(70) on an Intel-based Macintosh computer (http://neuron.yale.edu) and analyzed using Python scripts; further details are provided in Supplementary Methods.

Statistics. All data are given as mean \pm SEM. Paired or unpaired t-test was used to compare the mean of two groups when the data were normally distributed. Multiple groups were compared using ANOVA (one-, two- or three-factor) or repeated-measures ANOVA, depending on experimental setup; Bonferroni post-hoc test was used for comparison between groups; for data failing normality test Kruskal-Wallis ANOVA with Mann-Whitney post-hoc test was used. Statistical analyses were performed using IBM SPSS Statistics 21, GraphPad Prism or Origin. Statistical parameters are given in the figure legends.

Acknowledgments: We thank Prof Jim Deuchars and Dr Ronaldo Ichiyama (University of Leeds) for advice on experiments and helpful suggestions. We also thank Dr Junling Xing (Fourth Military Medical University) for help with setting up single unit recordings. This work was supported by the National Natural Science Foundation of China grants (84870872 \& 313400048), Key Basic Research Project of Applied Basic Research 
Program of Hebei Province (16967712D) and Science Fund for Creative Research Groups of Natural Science Foundation of Hebei Province (H2020206474) to X.D.; National Natural Science Foundation of China (91732108, 81871075) grants to H.Z.; Innovation fund for graduate students of Hebei Province (CXZZBS2018077) to H.H.; the Wellcome Trust Investigator Award 212302/Z/18/Z and Medical Research Council project grant (MR/V012738/1) to N.G.

Author Contributions N.G. and X.D. designed and directed the study; H.H., R.R., C.W., C.L., S.S., P.M., V.L., F.J., J.S. and N.X. conducted the experiments and analyzed data; D.B.J performed computational modeling; N.G., X.D. and H.H. wrote the paper, assisted by all the coauthors.

Competing interests: Authors declare that they have no competing interests

Data and materials availability: All data are included in the manuscript or Supplementary Materials; materials are available upon request

\section{References}

1. Melzack R, and Wall PD. Pain mechanisms: a new theory. Science. 1965;150(3699):971-9.

2. Braz J, Solorzano C, Wang X, and Basbaum AI. Transmitting pain and itch messages: a contemporary view of the spinal cord circuits that generate gate control. Neuron. 2014;82(3):52236.

3. Mendell LM. Constructing and deconstructing the gate theory of pain. Pain. 2014;155(2):210-6.

4. Duan B, Cheng L, Bourane S, Britz O, Padilla C, Garcia-Campmany L, et al. Identification of spinal circuits transmitting and gating mechanical pain. Cell. 2014;159(6):1417-32.

5. Barlow HB. In: Rosenblith W ed. Sensory Communication. M.I.T. Press; 1961:782-90.

6. Schmidt RF. In: Janzen R, Keidel, W.D., Herz, A., Steichele, C. ed. Pain. London: Churchill Livingstone; 1972:124-7.

7. Nathan PW. The gate-control theory of pain. A critical review. Brain. 1976;99(1):123-58.

8. Meyer RA, Raja SN, and Campbell JN. Coupling of action potential activity between unmyelinated fibers in the peripheral nerve of monkey. Science. 1985;227(4683):184-7.

9. Meyer RA, Raja SN, Campbell JN, Mackinnon SE, and Dellon AL. Neural activity originating from a neuroma in the baboon. Brain Res. 1985;325(1-2):255-60.

10. Amir R, and Devor M. Chemically mediated cross-excitation in rat dorsal root ganglia. $J$ Neurosci. 1996;16(15):4733-41.

11. Amir R, and Devor M. Functional cross-excitation between afferent A- and C-neurons in dorsal root ganglia. Neuroscience. 2000;95(1):189-95.

12. Kim YS, Anderson M, Park K, Zheng Q, Agarwal A, Gong C, et al. Coupled Activation of Primary Sensory Neurons Contributes to Chronic Pain. Neuron. 2016;91(5):1085-96.

13. Gemes G, Koopmeiners A, Rigaud M, Lirk P, Sapunar D, Bangaru ML, et al. Failure of action potential propagation in sensory neurons: mechanisms and loss of afferent filtering in C-type units after painful nerve injury. $J$ Physiol. 2013;591(Pt 4):1111-31.

14. Du X, Hao H, Gigout S, Huang D, Yang Y, Li L, et al. Control of somatic membrane potential in nociceptive neurons and its implications for peripheral nociceptive transmission. Pain. 2014;155(11):2306-22.

15. Du X, Hao H, Yang Y, Huang S, Wang C, Gigout S, et al. Local GABAergic signaling within sensory ganglia controls peripheral nociceptive transmission. J Clin Invest. 2017;127(5):174156.

16. Kent AR, Min X, Hogan QH, and Kramer JM. Mechanisms of Dorsal Root Ganglion Stimulation in Pain Suppression: A Computational Modeling Analysis. Neuromodulation. 2018;21(3):23446. 
17. Sundt D, Gamper N, and Jaffe DB. Spike propagation through the dorsal root ganglia in an unmyelinated sensory neuron: a modeling study. J Neurophysiol. 2015:jn 002262015.

18. Al-Basha D, and Prescott SA. Intermittent Failure of Spike Propagation in Primary Afferent Neurons during Tactile Stimulation. J Neurosci. 2019;39(50):9927-39.

19. Chao D, Zhang Z, Mecca CM, Hogan QH, and Pan B. Analgesic dorsal root ganglionic field stimulation blocks conduction of afferent impulse trains selectively in nociceptive sensory afferents. Pain. 2020.

20. Wall PD. Cord cells responding to touch, damage, and temperature of skin. J Neurophysiol. 1960;23:197-210.

21. Janig W, Schmidt RF, and Zimmermann M. Single unit responses and the total afferent outflow from the cat's foot pad upon mechanical stimulation. Exp Brain Res. 1968;6(2):100-15.

22. Obradovic AL, Scarpa J, Osuru HP, Weaver JL, Park JY, Pathirathna S, et al. Silencing the alpha2 subunit of gamma-aminobutyric acid type A receptors in rat dorsal root ganglia reveals its major role in antinociception posttraumatic nerve injury. Anesthesiology. 2015;123(3):65467.

23. Hanack C, Moroni M, Lima WC, Wende H, Kirchner M, Adelfinger L, et al. GABA blocks pathological but not acute TRPV1 pain signals. Cell. 2015;160(4):759-70.

24. Hoang TX, Nieto JH, Tillakaratne NJ, and Havton LA. Autonomic and motor neuron death is progressive and parallel in a lumbosacral ventral root avulsion model of cauda equina injury. $J$ Comp Neurol. 2003;467(4):477-86.

25. Koltzenburg M, Stucky CL, and Lewin GR. Receptive properties of mouse sensory neurons innervating hairy skin. J Neurophysiol. 1997;78(4):1841-50.

26. Braz JM, Sharif-Naeini R, Vogt D, Kriegstein A, Alvarez-Buylla A, Rubenstein JL, et al. Forebrain GABAergic neuron precursors integrate into adult spinal cord and reduce injuryinduced neuropathic pain. Neuron. 2012;74(4):663-75.

27. Etlin A, Braz JM, Kuhn JA, Wang X, Hamel KA, Llewellyn-Smith IJ, et al. Functional Synaptic Integration of Forebrain GABAergic Precursors into the Adult Spinal Cord. J Neurosci. 2016;36(46):11634-45.

28. Rigaud M, Gemes G, Barabas ME, Chernoff DI, Abram SE, Stucky CL, et al. Species and strain differences in rodent sciatic nerve anatomy: implications for studies of neuropathic pain. Pain. 2008;136(1-2):188-201.

29. Li L, Rutlin M, Abraira VE, Cassidy C, Kus L, Gong S, et al. The functional organization of cutaneous low-threshold mechanosensory neurons. Cell. 2011;147(7):1615-27.

30. Abraira VE, and Ginty DD. The sensory neurons of touch. Neuron. 2013;79(4):618-39.

31. Goldstein SS, and Rall W. Changes of action potential shape and velocity for changing core conductor geometry. Biophys J. 1974;14(10):731-57.

32. Luscher C, Streit J, Quadroni R, and Luscher HR. Action potential propagation through embryonic dorsal root ganglion cells in culture. I. Influence of the cell morphology on propagation properties. J Neurophysiol. 1994;72(2):622-33.

33. Debanne D, Campanac E, Bialowas A, Carlier E, and Alcaraz G. Axon physiology. Physiol Rev. 2011;91(2):555-602.

34. Ramón y Cajal S. Histologie du système nerveux de l'homme \& des vertébrés. Paris : Maloine; 1909.

35. Matsuda S, Kobayashi N, Terashita T, Shimokawa T, Shigemoto K, Mominoki K, et al. Phylogenetic investigation of Dogiel's pericellular nests and Cajal's initial glomeruli in the dorsal root ganglion. J Comp Neurol. 2005;491(3):234-45.

36. Ranson SW. The structure of the spinal ganglia and of the spinal nerves. J Comp Neurol. 1912;22:159-75.

37. Renier N, Adams EL, Kirst C, Wu Z, Azevedo R, Kohl J, et al. Mapping of Brain Activity by Automated Volume Analysis of Immediate Early Genes. Cell. 2016;165(7):1789-802.

38. Amir R, and Devor M. Electrical excitability of the soma of sensory neurons is required for spike invasion of the soma, but not for through-conduction. Biophys J. 2003;84(4):2181-91. 
39. Chai Z, Wang C, Huang R, Wang Y, Zhang X, Wu Q, et al. CaV2.2 Gates Calcium-Independent but Voltage-Dependent Secretion in Mammalian Sensory Neurons. Neuron. 2017;96(6):1317-26 e4.

40. Martens H, Weston MC, Boulland JL, Gronborg M, Grosche J, Kacza J, et al. Unique luminal localization of VGAT-C terminus allows for selective labeling of active cortical GABAergic synapses. J Neurosci. 2008;28(49):13125-31.

41. Galietta LJ, Haggie PM, and Verkman AS. Green fluorescent protein-based halide indicators with improved chloride and iodide affinities. FEBS Lett. 2001;499(3):220-4.

42. Johansson T, Norris T, and Peilot-Sjogren H. Yellow fluorescent protein-based assay to measure GABA(A) channel activation and allosteric modulation in CHO-K1 cells. PLoS One. 2013;8(3):e59429.

43. Namkung W, Yao Z, Finkbeiner WE, and Verkman AS. Small-molecule activators of TMEM16A, a calcium-activated chloride channel, stimulate epithelial chloride secretion and intestinal contraction. FASEB J. 2011;25(11):4048-62.

44. Shah S, Carver CM, Mullen P, Milne S, Lukacs V, Shapiro MS, et al. Local Ca ${ }^{2+}$ signals couple activation of TRPV1 and ANO1 sensory ion channels. Sci Signal. 2020;13(629).

45. Deer TR, Grigsby E, Weiner RL, Wilcosky B, and Kramer JM. A prospective study of dorsal root ganglion stimulation for the relief of chronic pain. Neuromodulation. 2013;16(1):67-72.

46. Liem L, Russo M, Huygen FJ, Van Buyten JP, Smet I, Verrills P, et al. A Multicenter, Prospective Trial to Assess the Safety and Performance of the Spinal Modulation Dorsal Root Ganglion Neurostimulator System in the Treatment of Chronic Pain. Neuromodulation. 2013; 16(5):47182.

47. Hunter CW, Sayed D, Lubenow T, Davis T, Carlson J, Rowe J, et al. DRG FOCUS: A Multicenter Study Evaluating Dorsal Root Ganglion Stimulation and Predictors for Trial Success. Neuromodulation. 2019;22(1):61-79.

48. Dun FT. The delay and blockage of sensory impulses in the dorsal root ganglion. $J$ Physiol. 1955;127(2):252-64.

49. Stoney SD, Jr. Unequal branch point filtering action in different types of dorsal root ganglion neurons of frogs. Neurosci Lett. 1985;59(1):15-20.

50. Tagini G, and Camino E. T-shaped cells of dorsal ganglia can influence the pattern of afferent discharge. Pflugers Arch. 1973;344(4):339-47.

51. Luscher C, Streit J, Lipp P, and Luscher HR. Action potential propagation through embryonic dorsal root ganglion cells in culture. II. Decrease of conduction reliability during repetitive stimulation. J Neurophysiol. 1994;72(2):634-43.

52. Debanne D. Information processing in the axon. Nat Rev Neurosci. 2004;5(4):304-16.

53. Wilke BU, Kummer KK, Leitner MG, and Kress M. Chloride - The Underrated Ion in Nociceptors. Front Neurosci. 2020;14:287.

54. Jager SE, Pallesen LT, Richner M, Harley P, Hore Z, McMahon S, et al. Changes in the transcriptional fingerprint of satellite glial cells following peripheral nerve injury. Glia. 2020;68(7):1375-95.

55. Usoskin D, Furlan A, Islam S, Abdo H, Lonnerberg P, Lou D, et al. Unbiased classification of sensory neuron types by large-scale single-cell RNA sequencing. Nat Neurosci. 2015;18(1):14553.

56. Tzeng HR, Lee MT, Fan PC, Knutson DE, Lai TH, Sieghart W, et al. alpha6GABAA Receptor Positive Modulators Alleviate Migraine-like Grimaces in Mice via Compensating GABAergic Deficits in Trigeminal Ganglia. Neurotherapeutics. 2021;18(1):569-85.

57. Lutter D, Ullrich F, Lueck JC, Kempa S, and Jentsch TJ. Selective transport of neurotransmitters and modulators by distinct volume-regulated LRRC8 anion channels. $J$ Cell Sci. 2017;130(6):1122-33.

58. Moore KA, Kohno T, Karchewski LA, Scholz J, Baba H, and Woolf CJ. Partial peripheral nerve injury promotes a selective loss of GABAergic inhibition in the superficial dorsal horn of the spinal cord. J Neurosci. 2002;22(15):6724-31. 
59. Llewellyn-Smith IJ, Basbaum AI, and Braz JM. Long-term, dynamic synaptic reorganization after GABAergic precursor cell transplantation into adult mouse spinal cord. J Comp Neurol. 2018;526(3):480-95.

60. Braz JM, Etlin A, Juarez-Salinas D, Llewellyn-Smith IJ, and Basbaum AI. Rebuilding CNS inhibitory circuits to control chronic neuropathic pain and itch. Prog Brain Res. 2017;231:87105.

61. Braz JM, Wang X, Guan Z, Rubenstein JL, and Basbaum AI. Transplant-mediated enhancement of spinal cord GABAergic inhibition reverses paclitaxel-induced mechanical and heat hypersensitivity. Pain. 2015;156(6):1084-91.

62. Hwang I, Hahm SC, Choi KA, Park SH, Jeong H, Yea JH, et al. Intrathecal Transplantation of Embryonic Stem Cell-Derived Spinal GABAergic Neural Precursor Cells Attenuates Neuropathic Pain in a Spinal Cord Injury Rat Model. Cell Transplant. 2016;25(3):593-607.

63. Yu H, Fischer G, Ebert AD, Wu HE, Bai X, and Hogan QH. Analgesia for neuropathic pain by dorsal root ganglion transplantation of genetically engineered mesenchymal stem cells: initial results. Mol Pain. 2015;11:5.

64. Liu Y, Weick JP, Liu H, Krencik R, Zhang X, Ma L, et al. Medial ganglionic eminence-like cells derived from human embryonic stem cells correct learning and memory deficits. Nat Biotechnol. 2013;31(5):440-7.

65. Manion J, Khuong T, Harney D, Littleboy JB, Ruan T, Loo L, et al. Human induced pluripotent stem cell-derived GABAergic interneuron transplants attenuate neuropathic pain. Pain. 2020;161(2):379-87.

66. Virtanen P, Gommers R, Oliphant TE, Haberland M, Reddy T, Cournapeau D, et al. SciPy 1.0: fundamental algorithms for scientific computing in Python. Nat Methods. 2020;17(3):261-72.

67. Chaure FJ, Rey HG, and Quian Quiroga R. A novel and fully automatic spike-sorting implementation with variable number of features. J Neurophysiol. 2018;120(4):1859-71.

68. Vogt D, Wu PR, Sorrells SF, Arnold C, Alvarez-Buylla A, and Rubenstein JL. Viral-mediated Labeling and Transplantation of Medial Ganglionic Eminence (MGE) Cells for In Vivo Studies. $J$ Vis Exp. 2015(98).

69. Liu B, Linley JE, Du X, Zhang X, Ooi L, Zhang H, et al. The acute nociceptive signals induced by bradykinin in rat sensory neurons are mediated by inhibition of $\mathrm{M}$-type $\mathrm{K}+$ channels and activation of Ca2+-activated Cl- channels. J Clin Invest. 2010;120(4):1240-52.

70. Hines ML, and Carnevale NT. NEURON: a tool for neuroscientists. Neuroscientist. 2001;7(2):123-35. 


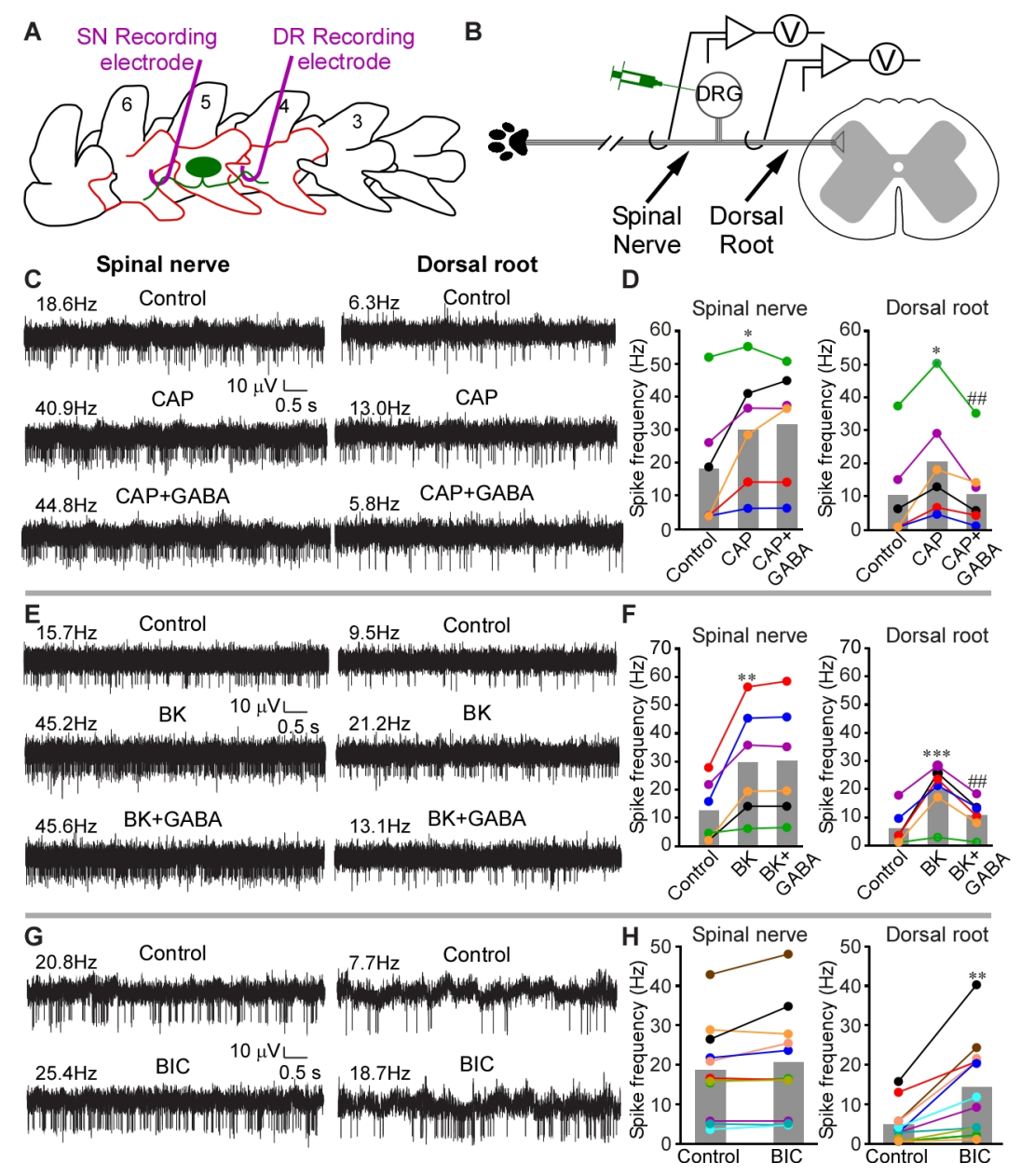

Figure 1. GABAergic filtering of nociceptive signals at the DRG. (A) Schematic of surgical exposure of the $L 5$ spinal nerve (SN, left), L5 DRG and the dorsal root (DR, middle) in an anesthetized rat. Parts of the vertebra that are surgically removed are shown in orange. SN and DR are then individually suspended on fine hook electrodes; DRG is exposed to direct drug application. (B), schematic of the electrode placement. (C) Hindpaw injection of Capsaicin (CAP, $10 \mu \mathrm{M}, 50 \mu \mathrm{l}$ ) increased firing frequency in both SN and DR branches of the nerve (middle traces, as compared to basal activity shown in the upper traces). Application of GABA ( $200 \mu \mathrm{M}, 3 \mu \mathrm{l}$ ) to DRG reduced CAP-induced firing frequency in DR but not SN (bottom traces). (D) Summary of panel C. Two-factor repeated measures ANOVA, with factors of nerve site (SN, DR) and drug treatment (Control, CAP, CAP+GABA) revealed main effects associated with nerve site $[F(1,10)=15.1$; $p<0.05]$ and drug application $[F(2,9)=12.8 ; p<0.05]$, and there was significant interaction between these factors $[F(2,9)=7.9 ; p<0.05]$. Bonferroni post-hoc test: *significant difference from control $(p<0.05)$; ${ }^{\#}$ significant difference from CAP ( $\left.p<0.01\right)$. (E), similar to $C$, but Bradykinin $(B K, 100 \mu M, 50 \mu l)$ was hindpaw injected, instead of CAP. (F) Summary of panel E. Two-factor repeated measures ANOVA: main effect associated with drug application $[F(2,9)=12.0 ; p<0.05]$; significant interaction between nerve site and drug application $[F(2,9)=11.5 ; p<0.05]$. Bonferroni post-hoc test: ${ }^{* *},{ }^{* * *}$ significant difference from control $(p<0.01$, $p<0.001)$; ${ }^{\prime}$ significant difference from BK ( $\left.p<0.01\right)$. (G) GABAA antagonist bicuculline (BIC, $\left.200 \mu \mathrm{M}, 3 \mu \mathrm{l}\right)$ was applied to DRG instead of GABA; hindpaw was not stimulated. (H) Summary of panel G. Two-factor repeated measures ANOVA: main effects associated with nerve site $[F(1,20)=7.7 ; p<0.05)$, drug application $[F(1,20)=12.0 ; p<0.01)$, significant interaction between nerve site and drug application $[F(1,20)=18.7$; $p<0.01]$. Bonferroni post-hoc test: ${ }^{* *}$ significant difference from control $(p<0.01)$. 


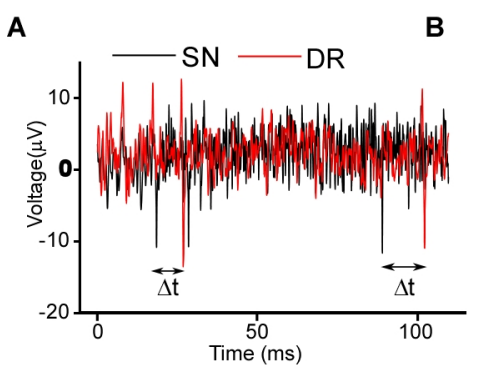

C
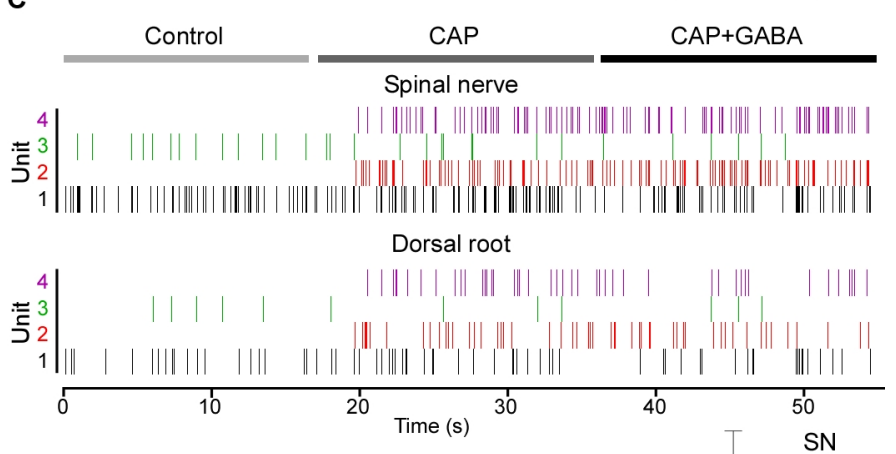

D

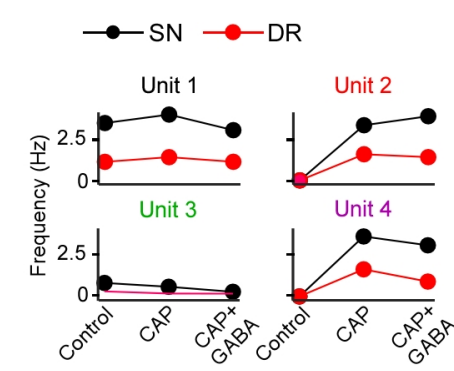

$\mathbf{F}$

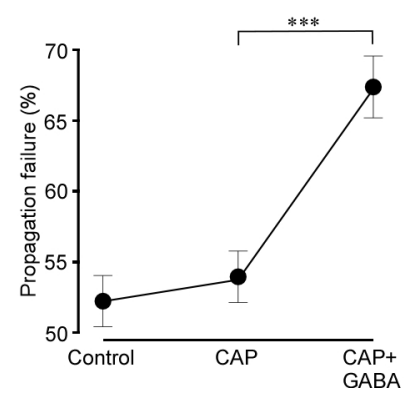

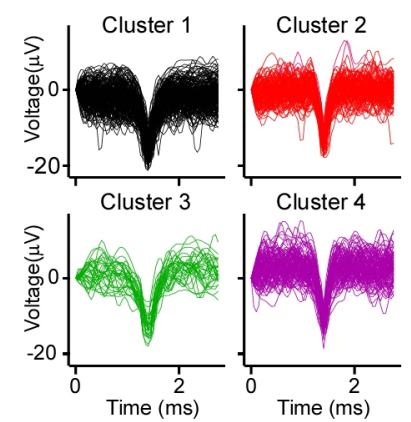

E

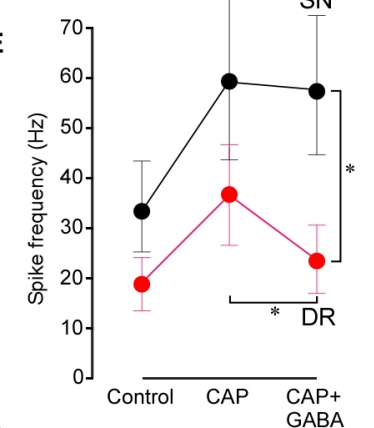

G

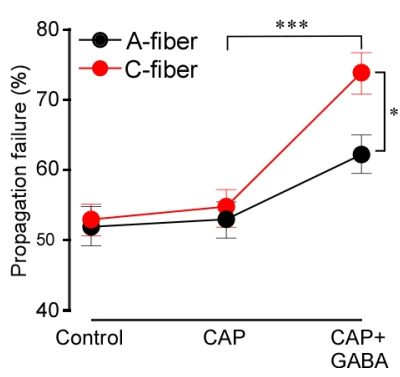

Figure 2. Spike analysis confirms GABAergic filtering. (A) Latencies between the $S N$ and DR spikes were measured; the minimum latency defined the SN origin of a DR spike. (B) Extracellular spike waveforms extracted from a spinal nerve recording and clustered using WaveClus. (C) Raster plot for each clustered waveform (denoted as Unit) under control, CAP and CAP+GABA conditions after matching the DR spikes with these in the SN. Note: capsaicin induced firing of specific units. (D) Firing rates of individual units identifies capsaicin-sensitive units. (E) Firing rates (matched spikes only) in SN and DR under control, CAP and CAP+GABA conditions. Two-factor (nerve site, drug application) repeated measures ANOVA: main effects associated with drug application $[F(2,10)=6.38 ; p<0.05]$, significant interaction between factors $[F(2,10)=10.74$; $p<0.01]$. Bonferroni post-hoc test: *significant difference from control $(p<0.05)$. $(F)$ Propagation failure $(\%)$ of all matched spike units under control, CAP and CAP+GABA conditions. One-way ANOVA: $F(2,117)=17.7$; $p<0.001$; Tukey post-hoc test: ${ }^{* * *}$ significant difference from CAP, $p<0.001$. (G) Units were divided into ' $C$-type' and 'A-type' based on the SN-DR latency $(C:<1.2 \mathrm{~m} / \mathrm{s} ; \mathrm{A}$ : $>1.2 \mathrm{~m} / \mathrm{s})$ and propagation failure rate analyzed as in (F). Two-factor (fiber type, drug application) mixed-effects ANOVA: main effect associated with drug 
application $[F(2,70)=34.82, p<0.001]$, significant interaction between factors $[F(2,72)=4.712, p<0.05]$. Sidak post-hoc test: *significant difference between $C A P+G A B A$ and CAP, significant difference between $A$ and $C$ fibers $(p<0.05)$. 

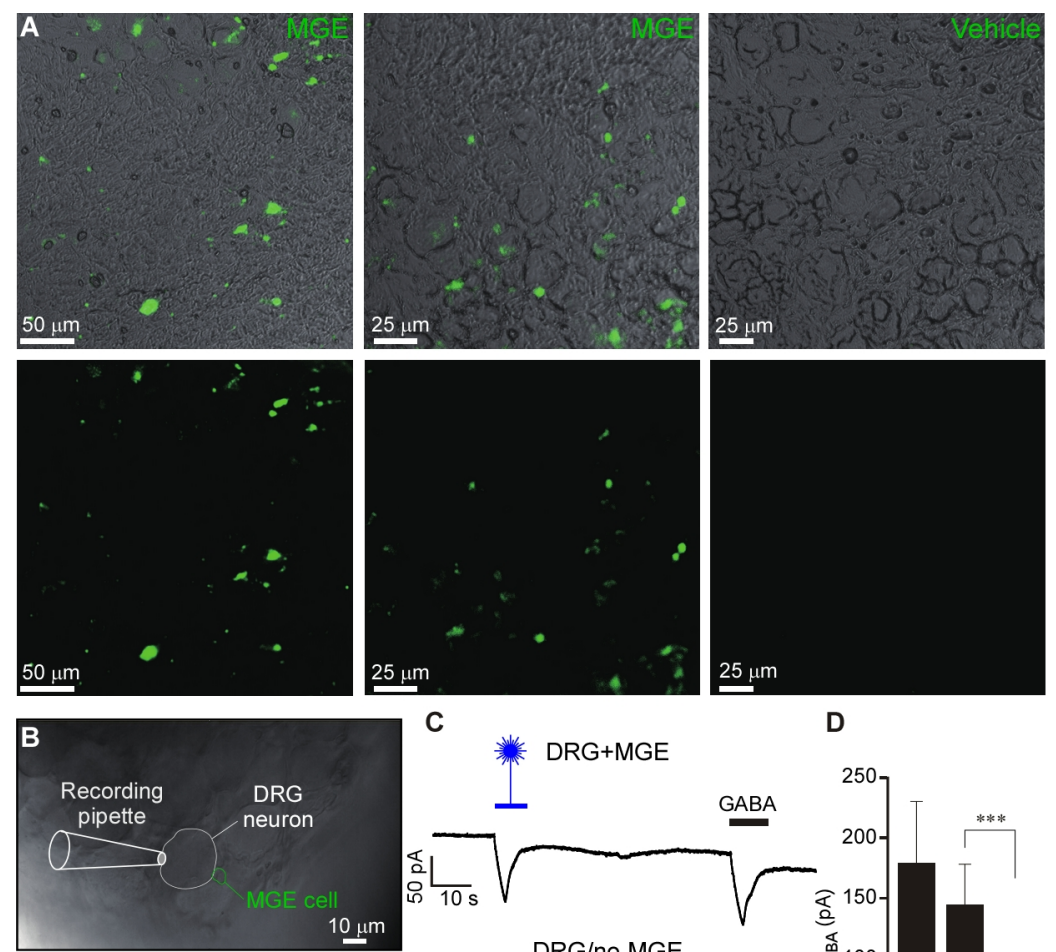

D

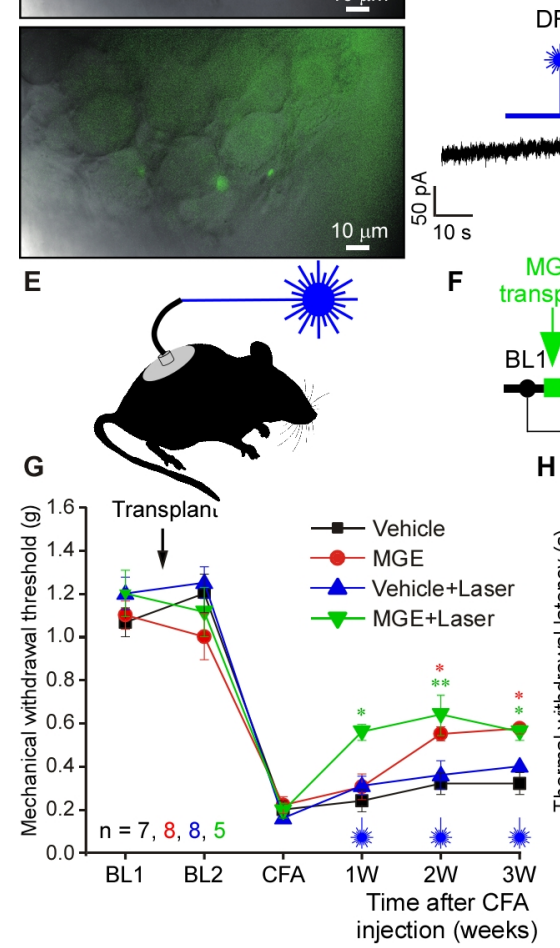

DRG/no MGE
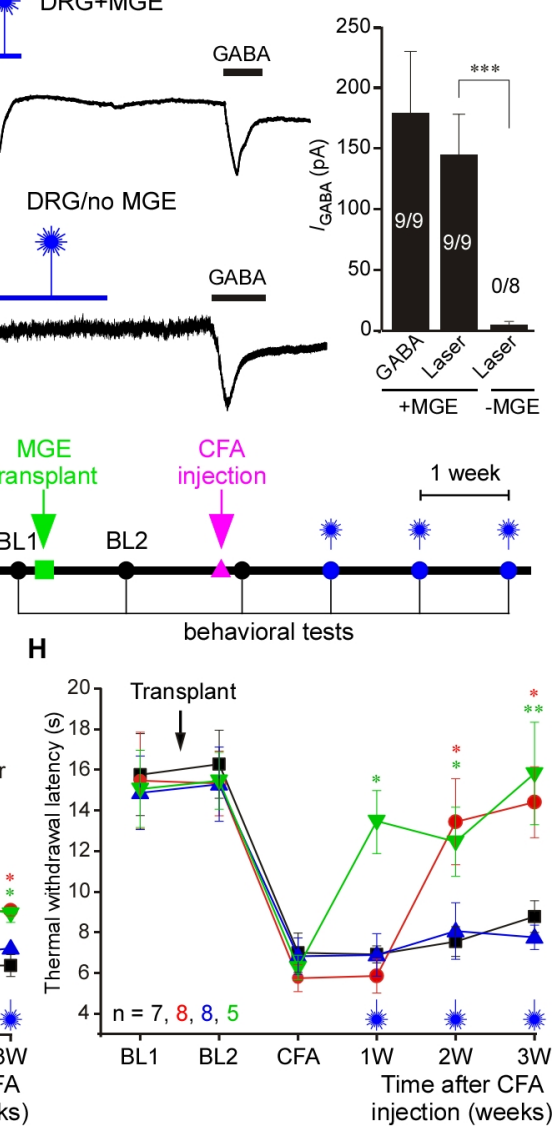

Figure 3. Transplantation of forebrain GABAergic neuron progenitor cells into the adult mouse DRG in vivo delivers an analgesic mechanism. (A) Fluorescence micrographs of DRG sections of mice 4 weeks after the injection with a suspension of medial embryonic eminence (MGE) cells derived fromVGAT-ChR2eYFP mice. Control mice (right images) received vehicle injections. (B) Bright-field and overlaid fluorescence images of 'loosened' DRG preparation used for patch clamp recording. The whole L4 DRG transplanted with VGAT-ChR2-eYFP MGE cells mice was extracted 4 weeks after transplantation. Recording was made from a DRG neuron juxtaposed to fluorescent MGE cell (top image) using whole-cell voltage clamp. (C) Top: example trace of continuous current recording $(-60 \mathrm{mV}$ ) from the cell shown in $\mathrm{B}$; stimulation with $473 \mathrm{~nm}$ blue laser (3 $\mathrm{mV}$ ) induced inward current, similar in amplitude and kinetics to the current induced by perfusion of GABA $(200 \mu \mathrm{M})$. Bottom: similar recording from a DRG of a vehicle-injected mice. (D) Summary for panel C; one-way 
ANOVA: $F(2,23)=5.9 ; p<0.01$; Bonferroni post-hoc test: ${ }^{* * *}$ significant difference between +MGE vs. $-\mathrm{MGE}$ $(p<0.001)$. Number of recorded/responsive cells is indicated within each bar. (E) Schematic of the in vivo optogenetic DRG stimulation. (F) Timeline of the in vivo behavioral testing after the MGE cell transplantation and hindpaw injection of CFA. (G, H) Mechanical $(G)$ and thermal $(H)$ hyperalgesia caused by hindpaw injection of CFA 2 weeks after MGE cells transplantation into L4 DRG of mice. At a time of MGE transplantation, mice were also implanted with the fiberoptic light guide. Mechanical and thermal sensitivity was measured using the von Frey and Hargreaves methods, respectively. Starting at one week after the CFA injection measurements were performed while stimulating the L4 DRG with 473nm laser. Black and blue symbols denote control mice DRG-injected with vehicle without and with optogenetic stimulation, respectively. Red and green symbols denote MGE-transplanted mice without and with optogenetic stimulation, respectively. BL1: baseline before transplantation; BL2: baseline after transplantation; CFA: 1 day after the plantar injection of CFA. G, Three-factor (MGE vs vehicle, time after CFA, laser stimulation) ANOVA: main effects associated with MGE transplantation $[F(1,24)=50.9 ; p<0.001]$, time after $\operatorname{CFA}[F(2,23)=6.6 ; p<0.01]$; laser stimulation $[F(1,24)=8.9 ; p<0.01]$. Bonferroni post-hoc test: red $^{*}$ indicate the difference between MGE group and vehicle group within the corresponding time point; green* indicate the difference between MGE with laser stimulation group and vehicle with laser stimulation group; ${ }^{*} p<0.05,{ }^{* *} p<0.01,{ }^{* * *} p<0.001$. H, Three-factor (MGE vs vehicle, time after CFA, laser stimulation) repeated measures ANOVA: main effects associated with MGE transplantation $[F(1,24)=37.4 ; p<0.001]$, time after $\operatorname{CFA}[F(2,23)=6.1 ; p<0.01]$, laser stimulation $[F(1,24)=2.4$; $p=0.12]$; significant interaction between time and laser stimulation $[F(2,23)=2.5 ; p=0.09]$ and between MGE and laser stimulation $[F(2,23)=3.2 ; p=0.08]$. Bonferroni post-hoc test: red $^{*}$ indicate the difference between MGE group and vehicle group within the corresponding time point; green* indicate the difference between MGE with laser stimulation group and vehicle with laser stimulation group; ${ }^{*} p<0.05,{ }^{* *} p<0.01,{ }^{* * *} p<0.001$. 
A

A Optogenetic

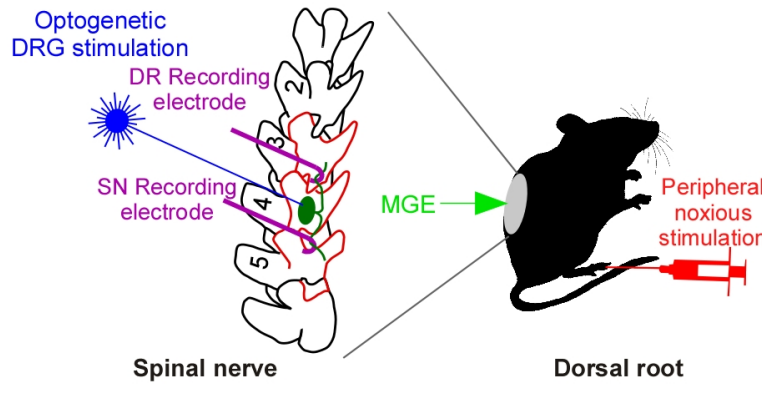

B Spinal nerve

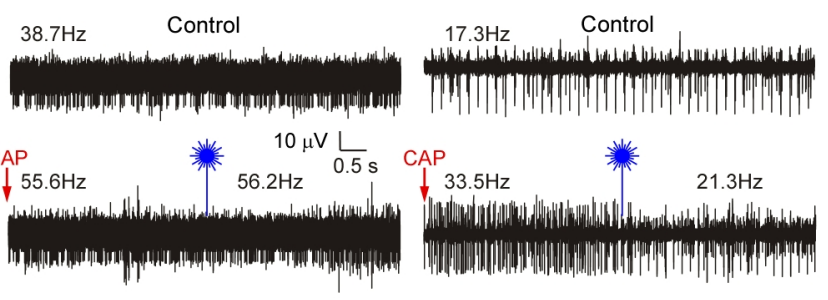

C

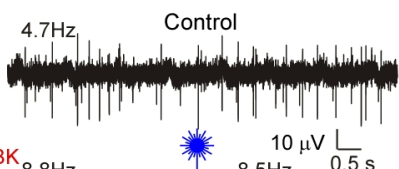

2.6Hz Control
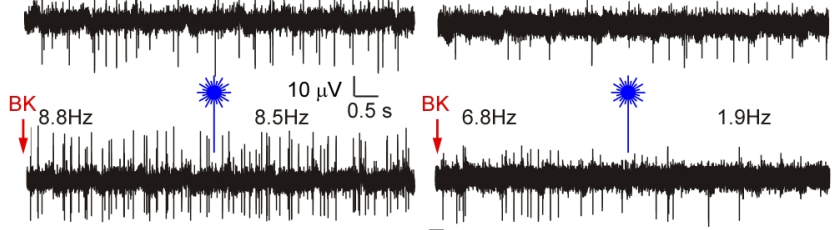

D

Spinal nerve

Dorsal root
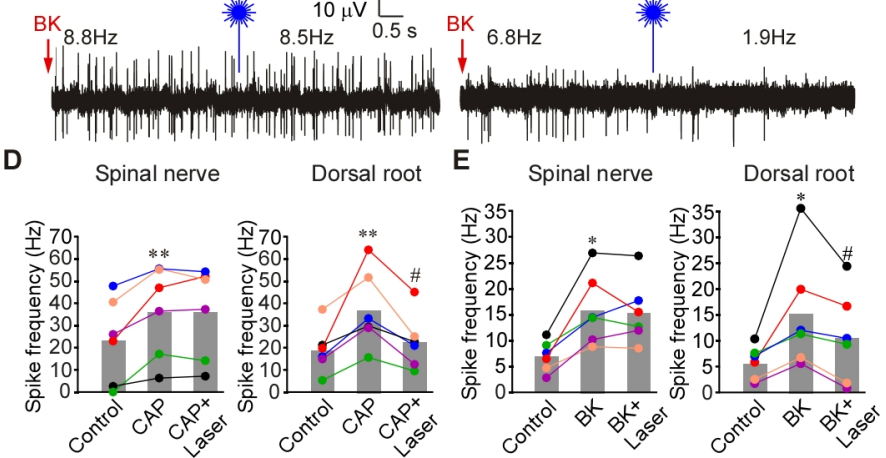

Figure 4. Optogenetic activation of MGE cells in DRG reduces frequency of firing induced by CAP or BK in the DR but not in the SN. (A) Schematic of the recording paradigm: mice were DRG-transplanted with VGAT-ChR2-eYFP MGE cells 3-4 weeks before recordings; recordings were done as shown in Fig. 1B, supplemented with optical stimulation of the exposed DRG. (B) Hindpaw injection of CAP (10 $\mu$ M, $20 \mu$; indicated by red arrow) increased firing frequency in both SN and DR. Application of $473 \mathrm{~nm}$ laser light to DRG (indicated by blue symbol) acutely reduced CAP-induced firing frequency in DR but not SN (bottom traces). (C) Experiment similar to A, but BK $(100 \mu \mathrm{M}, 20 \mu \mathrm{l})$ was hindpaw injected, instead of CAP. (D) Summary of panel B. Two-factor (nerve site, drug application) repeated measures ANOVA: main effect associated with drug treatment $[F(2,9)=11.4 ; p<0.05]$ and significant interaction between nerve site and treatment $[F(2,9)=7.6$; $p<0.05]$. Bonferroni post-hoc test: **significant difference from control $(p<0.01)$; \#significant difference from CAP (p<0.05). (E) Summary of panel C. Two-factor (nerve site, drug application) repeated measures ANOVA: main effect associated with nerve site $[F(1,10)=6.7 ; p<0.05]$ and significant interaction between nerve site and treatment $[F(2,9)=9.5 ; p<0.05]$. Bonferroni post-hoc test: *significant difference from control $(p<0.05)$; \#significant difference from BK $(p<0.05)$. 


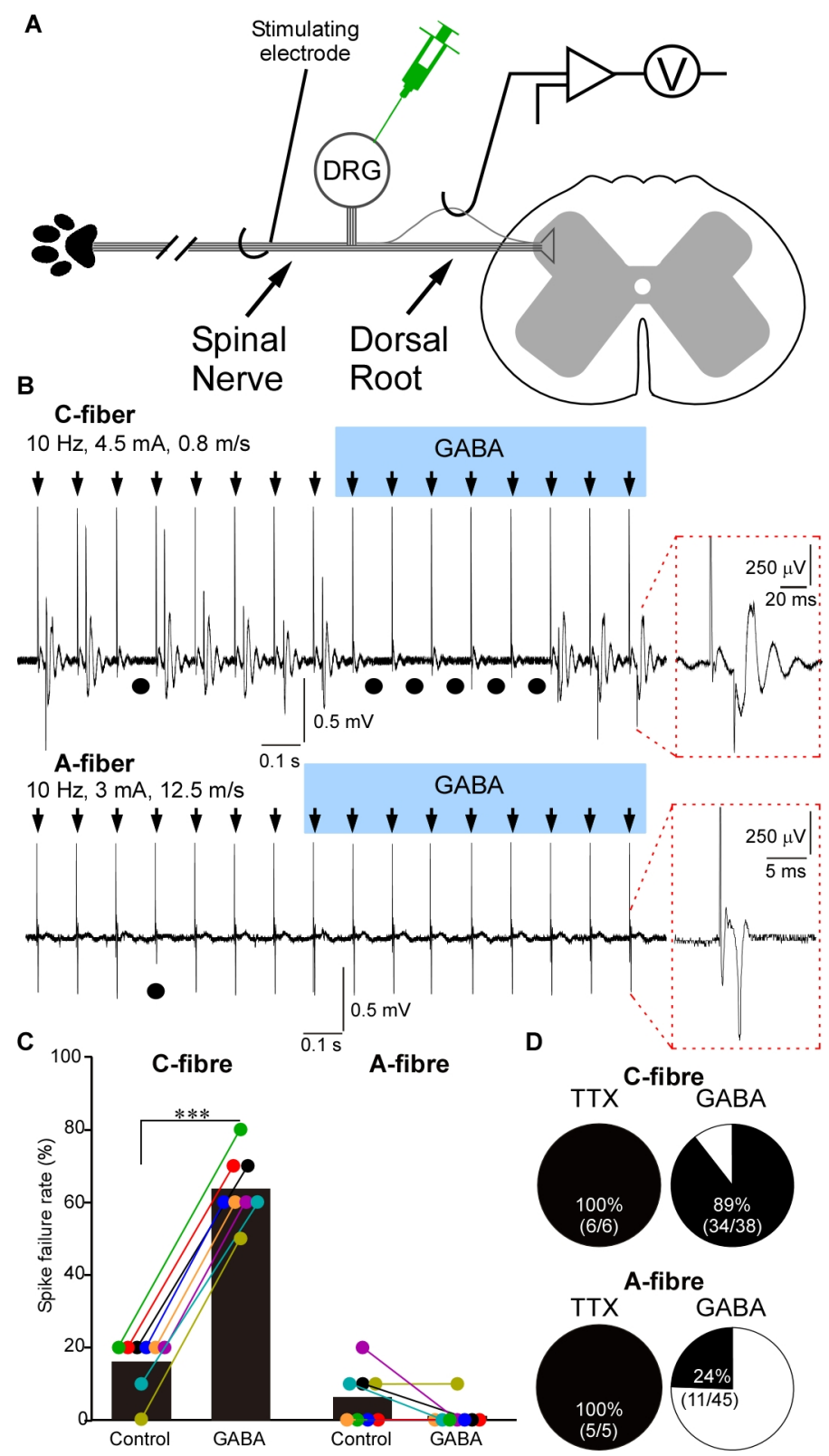

Figure 5. Single-unit recordings: DRG application of GABA induces spike failure at the DRG, which is specific to $\mathbf{C}$ fibers. (A) Schematic of the single unit recording paradigm. (B) Example traces of in vivo single unit recording from the DR process of a rat $\mathrm{C}$ fiber (top) or A fiber (bottom); stimulus electrode is placed in the spinal nerve. Parameters of stimulation and conduction velocity are indicated above the trace. GABA ( $200 \mu \mathrm{M}$, $3 \mu \mathrm{l}$ ) was applied to the DRG during period indicated by blue bar. Stimulus artifacts are indicated by black arrows. Black circles indicate failed spikes. Spike waveform is shown on the extended time scale on the right. (C). Spike failure rate before and during application of GABA is analyzed for $C$ and $A$ fibers (8-12 stimulations for each, the control period and during GABA application); $n=8$ for both fiber types; Two-factor (fiber type, drug application) repeated measures ANOVA: main effect associated with drug application $[F(1,28)=289.0$; $p<0.001]$ and significant interaction between fiber type and drug application $[F(1,28)=134.2 ; p<0.001]$. Bonferroni post-hoc test: "significant difference from control $(p<0.001)$. (D) Pie charts summarize the percentage of $\mathrm{C}$ and $\mathrm{A}$ fibers in which TTX or GABA produced a conduction block. Examples of TTX recordings are given in Suppl. Fig. 9. Number of experiments is indicated within each chart. 

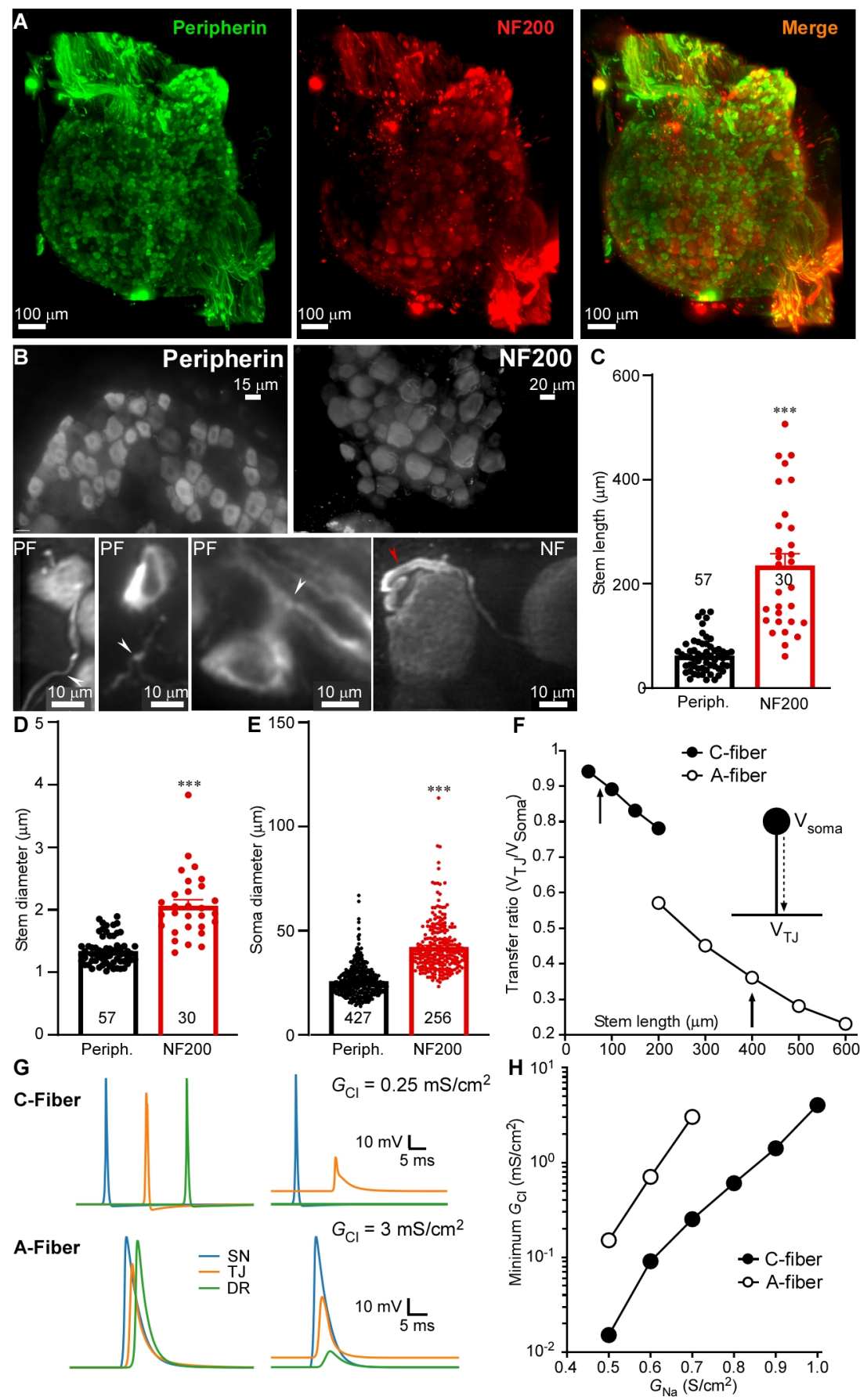

Figure 6. Initial axon morphology defines the efficiency of filtering in DRG. (A-B) Light-sheet microscopy of cleared rat DRG (see also Movie S1). C-fibers are labelled with peripherin and A-fibers are labelled with NF200. Stem axons were traced until bifurcation point using simple neurite tracer in FIJI (Movie S2). White arrowheads indicate t-junctions, red arrowhead point at glomerular region of A-fiber stem axon which increases the length. Quantification of the stem axon lengths (C), stem diameter (D) and somatic diameter (E) of the peripherin- and NF-200-labelled fibers is shown. Data are from 10 lumbar DRGs (peripherin) and 7 lumbar DRGs (NF200) from three rats; $n$ number is given within each bar. Unpaired t-test: $t(85)=9.8, p<0.001$ (C); $t(85)=9.8, p<0.001(D) ; t(681)=21.6, p<0.001(E)$. Per-animal analysis of data in C-F are shown in Suppl. Fig. 10. (F-H) Biophysical model of rat A-type fiber. (F) Voltage transfer ratio (potential at the t-junction produced by a potential generated at the soma) as a function of stem length. For A-fibers, this was achieved by varying the number of internode/node pairs. Arrows indicate the typical stem lengths used when simulating spike propagation through the DRG. (G) In the absence of somatic $G_{c I}$ (left traces), spike elicited in the spinal nerve 
(SN) section of the model propagate through the t-junction (TJ) and into the dorsal root (DR) in both C-fiber and A-fiber models. With a somatic $\mathrm{G}_{\mathrm{Cl}}$ (right traces), the threshold for blockade in $\mathrm{C}$-fibers is lower. Note that greater $\mathrm{G}_{\mathrm{cl}}$ in the A-fiber model produces less depolarization at the soma compared with the C-type model. $(\mathbf{H})$ Minimum somatic $\mathrm{G}_{\mathrm{Cl}}$ for blocking spike propagation is plotted against $\mathrm{Na}^{+}$conductance $\left(\mathrm{G}_{\mathrm{Na}}\right)$. For $\mathrm{G}_{\mathrm{Na}}$ greater than $0.7 \mathrm{mS} / \mathrm{cm}^{2}$, somatic $\mathrm{G}_{\mathrm{cl}}$ was unable to block spike propagation. 

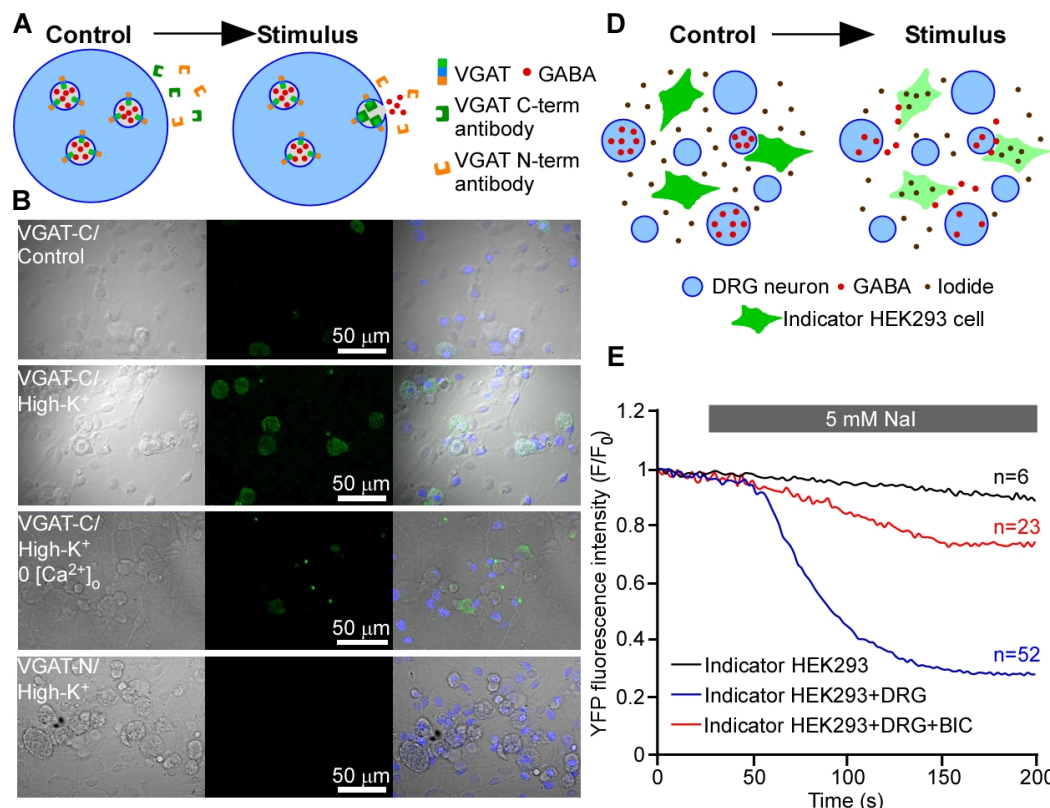

DRG neuron • GABA • lodide

$\checkmark$ Indicator HEK293 cell

E
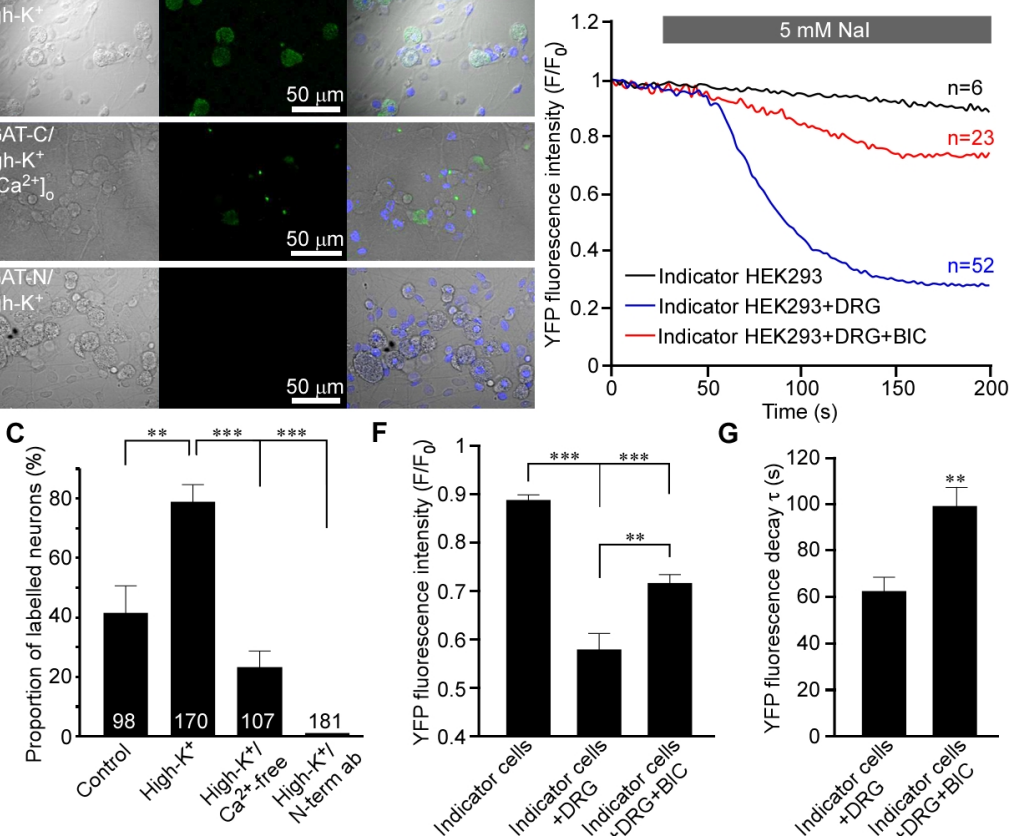

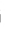

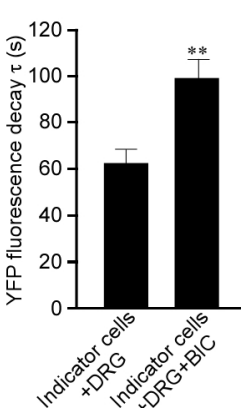

Figure 7. Activity-dependent and spontaneous GABA release of GABA from DRG neurons. (A-C) Vesicular GABA release from DRG neurons studied with luminal VGAT antibody uptake. (A) Schematic of activity-dependent uptake of luminal (C-terminal) VGAT antibody. (B) Bright-field (left), fluorescence (middle) and overlaid images of DRG culture incubated in the presence of either a luminal VGAT antibody (C-term; three upper rows) or a cytoplasmic (N-term; bottom row) antibody. Cells were incubated in the control extracellular solution (upper row) or depolarized by extracellular solution containing $100 \mathrm{mM} \mathrm{KCl}$ (second from the top and the bottom rows). In the second from the bottom row $\mathrm{Ca}^{2+}$ was excluded from the extracellular solution. (C) Summary of experiments like these shown in $B(N=3-5$, number of neurons is indicated within the columns); quantified is the percentage of stained neurons ${ }^{* *} p<0.01,{ }^{* * *} p<0.01$ (Fisher's exact test with Bonferroni correction). (D-G) GABA release from DRG investigated with the use of indicator HEK293 cells. (A) Schematic of the experiment: HEK293 cells transfected with $\alpha 1, \beta 2$ and $\gamma 2$ subunits of $\mathrm{GABA}_{\mathrm{A}}$ receptors and a halide-sensitive EYFP mutant (H148Q/I152L; EYFP-QL) are co-cultured with DRG neurons and imaged in the presence of $5 \mathrm{mM}$ extracellular iodide. When GABA is present in the extracellular solution, GABAA channel opening causes I- influx and EYFP-QL fluorescence quenching. (E) Application of Nal induced bicucullinesensitive EYFP-QL quenching only when indicator HEK293 cells are co-cultured with DRG but not in monoculture; representative traces are shown for each experimental condition. (F) Summary for panel E; normalized fluorescence intensity is quantified at $200 \mathrm{~s}$ of each recording. Kruskal-Wallis $A N O V A$ : $H(2)=33.6$, $\mathrm{p}<0.001$; Mann-Whitney post-hoc test: significant difference between indicated groups, ${ }^{* *} p<0.01,{ }^{* * *} p<0.001$ (G) Quantification of the kinetics of the EYFP-QL fluorescence quenching in experiments shown in E; individual recordings were fit with exponential function and time constants $(\tau)$ of the fluorescence decay were analyzed. 
Mann-Whitney test: $U=786, p<0.01$. In E-F, data from three independent experiments for each condition are shown, number of cells analyzed is indicated above each trace in panel $\mathrm{E}$. 